\title{
ABSTRACT
}

\section{CONSTRUCTION OF EFFECTIVE ELECTROMAGNETIC CURRENTS FOR TWO-BODY QUASIPOTENTIAL EQUATIONS}

\author{
Dmitri Krioukov \\ Old Dominion University, 1998 \\ Director: Dr. J. W. Van Orden
}

A systematic algebraic approach for the construction of effective electromagnetic currents consistent with relativistic two-body quasipotential equations is presented. This approach generalizes the Mandelstam formalism and applies it to a generic quasipotential reduction method. The use of WardTakahashi identities for the effective currents guarantees conservation of current matrix elements involving any combination of bound and scattering states. This approach is shown to reproduce previous results for current matrix elements for the particular cases of the Gross and Blankenbecler-Sugar equations. A generic method of truncation of the quasipotential effective current with respect to the number of boson exchanges is introduced. 


\title{
CONSTRUCTION OF EFFECTIVE ELECTROMAGNETIC CURRENTS FOR TWO-BODY QUASIPOTENTIAL EQUATIONS
}

\author{
by \\ Dmitri Krioukov \\ Diploma in Physics, February 1993, St.Petersburg University \\ A Dissertation submitted to the Faculty of \\ Old Dominion University in Partial Fullfillment of the \\ Requirement for the Degree of \\ DOCTOR OF PHILOSOPHY \\ PHYSICS \\ OLD DOMINION UNIVERSITY \\ September 1998
}

Approved by:

J. W. Van Orden (Director) 
(C) Copyright by

Dmitri Krioukov

1998

All Rights Reserved 


\section{Acknowledgements}

I would like to thank Dr. Van Orden for his comprehensive support in my Ph.D research project. I greatly appreciated his readiness to find time to communicate even in the circumstances that were very difficult for himself. His help in solution of the computer equipment problem enormously facilitated the creation of this text.

I am also grateful to all members of my dissertation committee in general and to Dr. Radyushkin in particular for their time reading this text and making valuable comments.

I also thank all other members of the Physics Department of ODU and especially Dr. Hyde-Wright and Mr. Hooks for the warm and friendly atmosphere I have enjoyed so much these four years. 


\section{Table of Contents}

List of Tables $\quad$ v

List of Figures vii

1 Introduction 1

2 Bethe-Salpeter-Mandelstam Formalism $\quad 7$

2.1 Four-point Propagator . . . . . . . . . . . . . 7

2.2 Scattering Matrix and Unitarity . . . . . . . . . . . . 11

2.3 Bound State and Wave Equation . . . . . . . . . . . . 13

2.4 Normalization Condition . . . . . . . . . . . . 16

2.5 Current Operator and Five-point Function . . . . . . . . . 17

2.6 Charge Conservation ................ 21

3 Quasipotential Formalism $\quad 23$

3.1 Motivations and General Requirements . . . . . . . . . . 23

3.1.1 Motivations and Requirements . . . . . . . . 24

3.1.2 Review of Proposed Quasipotential Propagators . . . . 27

3.2 Generic Approach . . . . . . . . . . . . . . . . . 31

3.2.1 Four-point Propagator ............. 31

3.2.2 Current Operator and Five-point Function . . . . . . 35

3.2.3 Current Conservation . . . . . . . . . . . 37

3.2.4 Current Matrix Elements and Charge Conservation . . 41 
4 Examples $\quad 44$

4.1 Blankenbecler-Sugar Case .............. 44

4.2 Gross Case ...................... 52

5 Truncation Issues $\quad 63$

5.1 Bethe-Salpeter Case ................. 64

5.2 Generic Quasipotential . . . . . . . . . . 66

5.2.1 Kernel Expansion . . . . . . . . . . . 66

5.2 .2 Current Expansion .............. 68

5.2 .3 Current Truncation . . . . . . . . . . . 74

5.2 .4 OBE Example.................. 82

6 Conclusion and Discussions $\quad 85$ 


\section{List of Tables}

1 Proposed families of quasipotential propagators. . . . . . . . 29

$2(204) \mapsto(205)$ transformation. . . . . . . . . . . 73 


\section{List of Figures}

1 Reducible diagrams. ................. 8

2 Two-body irreducible Bether-Salpeter kernel. . . . . . . . . 8

3 Four-point propagator. .................. 9

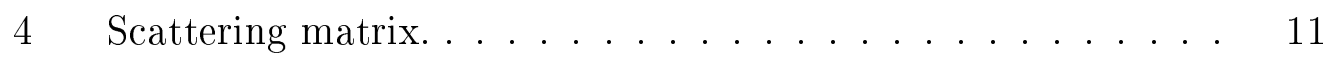

5 Bethe-Salpeter equation. ................ 11

6 Bound state. . . . . . . . . . . . . 13

7 Bethe-Salpeter equation for the vertex function. . . . . . . . 14

8 Current operator. .................... 18

9 Five-point function. . . . . . . . . . . . . . 19

10 Phase space reduction. . . . . . . . . . . . . 32

11 The IA part of the BBS current for the first particle. . . . . . 48

12 The quasipotential equation for $\mid \Gamma>\ldots . . . . . . . .448$

13 The quasipotential equation for $<\Gamma \mid$. . . . . . . . . 48

14 The IA part of the Gross current for the first particle. . . . . . 55

15 The IA part of the Gross current for the second particle. . . . 57

16 Current $J_{\mathrm{GM}, 2}^{\mu} \ldots \ldots \ldots \ldots \ldots \ldots \ldots$

17 In any off-shell case, the current can not be reduced. . . . . . 60

18 The original Gross effective current. . . . . . . . . . . . 61

19 The quasipotential kernel series. . . . . . . . . . . 67

20 The divergence and expansion of the effective current. . . . . 68

21 No truncation. . . . . . . . . . . . . 76

22 The $n$-boson exchange contributions. . . . . . . . . 76 
23 Up-to- $N$-boson exchange truncation. . . . . . . . . 78

24 Current is conserved. . . . . . . . . . . . 79

25 The interpretation of the current $J_{Q}^{\mu[N]} \ldots \ldots . . . . . \quad 80$ 


\section{Chapter 1}

\section{Introduction}

The theory of bound and low energy scattering states is one of the most actively studied fields in few-body physics. Historically, the non-relativistic approach in the form of the Lippman-Schwinger [1] equation (which is basically the Schrödinger equation rewritten as an integral equation in the momentum space) was the most popular method used for the description of twobody systems. The Lippman-Schwinger equation is the primary result for the non-relativistic scattering on an analytically "good" potential $V$, and it was thought to be a reasonably fair tool to study a low energy two-body system. However, the necessity of the relativistic approach has also been long recognized. Recent experiments in the Thomas Jefferson National Accelerator Facility (formerly CEBAF) [2] on the elastic scattering and the photodisinte-

gration of the deuteron are examples of a number of dramatic indications of the importance of the relativistic effects

in this theory. During these experiments, high momentum transfers were observed. They lead to essentially relativistic behavior of a composite target and the inevitability of the recourse to relativistically covariant models 
becomes obvious.

If a two-body system can form a bound state, then, in both the relativistic and non-relativistic scattering theories, this bound state corresponds to a pole in the scattering matrix. In the relativistic theory, this pole does not exist in any Feynman diagram or any finite set of Feynman diagrams defining the scattering matrix. Only the infinite number of diagrams (i.e. the scattering matrix itself) has the pole. Since it is impossible to consider the infinite number of all Feynman diagrams, some subset of diagrams, which are believed to be physically important for a model in question, is taken into account. A model of two heavy nucleons interacting by exchanges of a light boson (along with the assumption that all self-energy and vertex correction diagrams are already included or ignored or can be treated by external methods) leads to the subset of the infinite number of diagrams that can be expressed by means of an integral equation. This equation is called the Bethe-Salpeter [3] equation for the scattering matrix and it was the first relativistically covariant result in the theory of two-body systems.

As soon as one relativistically covariant equation is found, it is not difficult to see [4] that there exists an infinite spectrum of other relativistic integral equations that produce exactly the same solution as (are equivalent to) the Bethe-Salpeter equation. These equations are called the quasipotential equations and there are at least two reasons not to limit the theory to studying only one representative (the Bethe-Salpeter equation) of this set of equations.

First, it should be emphasized that the Bethe-Salpeter equation can be solved exactly [5]. However, the exact calculations using the FeynmanSchwinger representation [6] were performed only for the scalar case, and it is not clear if these calculations use a feasible amount of the computer time. 
On the other hand, Kusaka et al. [7] developed a method allowing them to calculate the Bethe-Salpeter vertex function for an arbitrary scattering kernel. However, the exact value of the Bethe-Salpeter kernel, which incorporates the infinite number of contributions, is not known and, hence, the usual practice is to truncate the kernel at some number of boson exchanges before numerical solutions $[8,9,10,11]$ can be sought. Being truncated at the lowest order, the Bethe-Salpeter approach produces less satisfactory solutions than any of the quasipotential equations $[1,5]$. This is hardly unexpected since there is no reason to assume that the Bethe-Salpeter equation, being truncated, provides for the best description of the two-body system.

Second, all quasipotentials are characterized by the reduction the fourdimensional momentum space available for the system in the Bethe-Salpeter case to some three-dimensional submanifold. This drastically simplifies numerical calculations [1]. More detailed consideration of these two issues is done in Section 3.

Of course, one of the most important topics in this theory is electromagnetic scattering. The electromagnetic effective current is a central object here. Its matrix elements on bound or scattering states describe the electromagnetic properties of the system. The construction of the effective current in the Bethe-Salpeter case is a very stream-lined procedure and is based upon the fundamental field theoretical facts about the relation between the $n$-point propagators and the Bethe-Salpeter bound and scattering wave functions. These results were first observed by Mandelstam in [12]. The fact that the current constructed this way is conserved is checked by the active use of the WardTakahashi [13] identities. This was most straightforwardly illustrated by Bentz in $[14]$. 
Unfortunately, there has been no systematic approach to the construction of the effective current operator for the generic quasipotential approach. This was partly related to the absence of a clear quasipotential formalism analogous to the strict field theoretical formalism suggested by Mandelstam for the Bethe-Salpeter case. Although the first attempts to introduce this formalism were made in $[15,16]$, the results obtained there could not be considered final since the primary purposes of the authors were different. The authors of [16] made a very careful analytical consideration of all loop integrals in the Gross case. This allowed them to give a reasonable definition of the $n$-point point propagators and the effective current, which turned out to follow directly from results obtained in this text based upon different principles. On the other hand, the underlying fundamentals of the quasipotential effective current definition in [15] (equation (16)) were not clear; and, as a result, the current obtained there did not satisfy the obvious requirements we discuss in Section 6 (the last example we consider in Section 6 is the current obtained in $[15])$.

Starting from the fact that the physical observables can not change whatever quasipotential or the Bethe-Salpeter approach is in effect and using this as a fundamental principle, we define the quasipotential $n$-point propagators. We demonstrate then that, at the algebraic level, the quasipotential formalism looks exactly as Mandelstam's formalism in the Bethe-Salpeter case, which means that the Mandelstam formalism can be extended to any quasipotential approach given that this quasipotential approach satisfies some requirements and is characterized by some additional properties discussed in Section 3.1.1. The specifics of one or another three-dimensional reduction 
technique do not matter, and even the equal-time quasipotential approach introduced by Phillips and Wallace [17] can be described in terms of this new formalism (cf. the discussion at the end of Section 3.1).

The Bethe-Salpeter case still plays its exceptional role: the quasipotential $n$-point propagators are no longer the Fourier transforms of the vacuum expectation values of the time ordered products of the corresponding nucleon field operators. Instead, some integral equations are established to relate the quasipotential $n$-point propagators to those in the Bethe-Salpeter case. As soon as these equations are solved, the quasipotential approach becomes equivalent to the Bethe-Salpeter approach. Obviously, this should be of no surprise since the equivalence is embedded into the theory from the very beginning. The procedure described above allows us to write a generic expression for the effective current operator which reproduces existing results in particular cases.

The generic effective current introduces some difficulties in the truncation techniques. The problem there is associated with the current non-linearity with respect to the quasipotential kernel and the exchange current that are to be truncated. We work out a prescription for how the effective current should be truncated in order to retain current conservation.

In Section 2, we remind the reader of the basic Bethe-Salpeter-Mandelstam formalism. Section 3 is devoted to the quasipotential approach. Section 3.1 contains the fundamental requirements for the quasipotential propagator, which defines the quasipotential approach as a whole. We briefly review some of the quasipotential approaches proposed and considered in the literature and present a new superfamily of quasipotential propagators. In Section 3.2, we introduce the quasipotential Mandelstam formalism mentioned above. Section 4 deals with the two most famous examples, the Blankenbecler-Sugar and Gross 
quasipotentials. We show how the generic effective current is reduced to the existing results in these particular cases. Section 5 fixes the problems associated with truncation in the generic quasipotential case. Section 6 contains some conclusions and discusses some results. The material in Sections 3.2, 4, 5.2, and the final comments in Section 6 are new.

When we have to use an explicit form of propagators, we confine ourselves to the scalar $\phi^{3}$ theory to avoid unnecessary complication of the formulae. However, we still call the bound (or scattering) state constituents either fermions or nucleons. 


\section{Chapter 2}

\section{Bethe-Salpeter-Mandelstam Formalism}

The Bethe-Salpeter equation plays a central role in the relativistic approach to the theory of bound and scattering states. It represents a quantum field theory result for the scattering amplitude, $\mathcal{M}$. A rigorous derivation of the Bethe-Salpeter equation can be found elsewhere $[3,18,19]$. In this section, we reproduce the basic steps leading to the formulae we will further refer to and call the Mandelstam formalism.

\section{$2.1 \quad$ Four-point Propagator}

All Feynman diagrams representing a system of two "nucleons" (solid lines on all subsequent figures) interacting by "meson" (dashed lines) exchanges are divided into two classes. Some representatives of the first class are shown on Fig. 1. Those diagrams are said to be reducible since they can be reduced, i.e. split into the product of several simpler diagrams by cutting just nucleon 

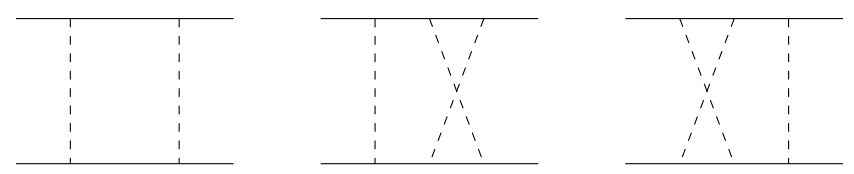

Figure 1: Reducible diagrams.

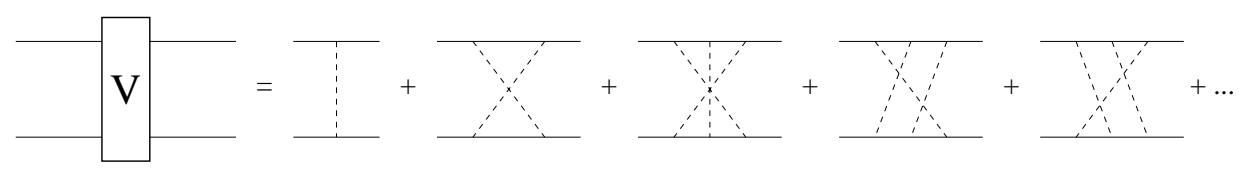

Figure 2: Two-body irreducible Bether-Salpeter kernel.

lines.

The two-body irreducible Bethe-Salpeter kernel, $V$, is then defined as an infinite sum of all members of the second class of diagrams called, as expected, irreducible. This sum (with up to three boson exchanges) is presented in Fig. 2.

The free two-body propagator, $G_{B S}$, is defined as a product of the two single-particle propagators, $G_{i} ; i=1,2$. If we introduce the following notations:

$$
\begin{aligned}
P & =p_{1}+p_{2}, \\
p & =\frac{1}{2}\left(p_{1}-p_{2}\right),
\end{aligned}
$$

for the total and relative 4-momenta of the two-particle system respectively, then we have that

$$
G_{B S}(p ; P)=-i G_{1}\left(\frac{1}{2} P+p\right) G_{2}\left(\frac{1}{2} P-p\right)
$$

At this point we can write down an exact result from the quantum field theory for the four point propagator, $\mathcal{G}$, which is the solution of the following 


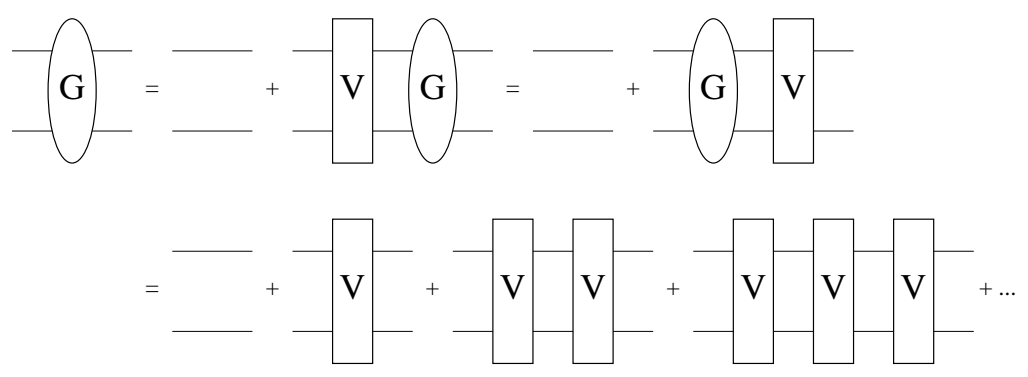

Figure 3: Four-point propagator.

integral equation:

$$
\begin{aligned}
\mathcal{G}\left(p^{\prime}, p ; P\right) & =(2 \pi)^{4} \delta^{4}\left(p^{\prime}-p\right) G_{B S}(p ; P) \\
& -\int \frac{d^{4} k}{(2 \pi)^{4}} G_{B S}\left(p^{\prime} ; P\right) V\left(p^{\prime}, k ; P\right) \mathcal{G}(k, p ; P),
\end{aligned}
$$

or, equivalently,

$$
\begin{aligned}
\mathcal{G}\left(p^{\prime}, p ; P\right) & =(2 \pi)^{4} \delta^{4}\left(p^{\prime}-p\right) G_{B S}(p ; P) \\
& -\int \frac{d^{4} k}{(2 \pi)^{4}} \mathcal{G}\left(p^{\prime}, k ; P\right) V(k, p ; P) G_{B S}(p ; P),
\end{aligned}
$$

where $P, p$ and $P^{\prime}, p^{\prime}$ stand for the total and relative momenta in the initial and final states respectively.

Equations (4) and (5) express the fact that the four-point propagator is an infinite series of Feynman diagrams with all possible boson exchanges between the two particles. This fact is readily illustrated by Fig. 3, given that we already have a graphical representation of kernel $V$ on Fig. 2.

Indeed, consider the second row of Fig. 3. It can be obtained by successive iterations of the basic relations (4) or (5) shown on the first row of Fig. 3. If we now approximate $V$ by just its first term from Fig. 2, then $\mathcal{G}$ will be equal to the sum of all ladder diagrams. If we also count the second diagram from the 
right hand side of Fig. 2, then the building blocks of the infinite series on the second row on of Fig. 3 will consist of the box and cross-box diagrams. Finally, if we take the precise result for $V$ (an infinite sum), then the second row on Fig. 3 will obviously consist of all possible ladder and cross-ladder diagrams contributing to the interaction between the two particles.

From now on, to save the space and for simplicity, we will try not to use the integral form of equations we write and, where possible, our preference will be given to the operator form of all resulting expressions.

To rewrite, for example, equation (4) in its operator form, we have to introduce operators $V, \mathcal{G}$ and $G_{B S}$ for the Bethe-Salpeter kernel, four-point propagator and the free two-body propagator respectively. They can be defined by their matrix elements in momentum space of the two particles:

$$
\begin{aligned}
<p_{1}^{\prime} p_{2}^{\prime}|V| p_{1} p_{2}>= & (2 \pi)^{4} \delta^{4}\left(P^{\prime}-P\right) V\left(p^{\prime}, p ; P\right), \\
<p_{1}^{\prime} p_{2}^{\prime}|\mathcal{G}| p_{1} p_{2}>= & (2 \pi)^{4} \delta^{4}\left(P^{\prime}-P\right) \mathcal{G}\left(p^{\prime}, p ; P\right), \\
<p_{1}^{\prime} p_{2}^{\prime}\left|G_{B S}\right| p_{1} p_{2}>= & -i(2 \pi)^{4} \delta^{4}\left(P^{\prime}-P\right)(2 \pi)^{4} \delta^{4}\left(p^{\prime}-p\right) \\
& \times G_{1}\left(\frac{1}{2} P+p\right) G_{2}\left(\frac{1}{2} P-p\right),
\end{aligned}
$$

where the following assumptions about the momentum space states are implied:

$$
\begin{aligned}
<p^{\prime} \mid p> & =(2 \pi)^{4} \delta^{4}\left(p^{\prime}-p\right), \\
1 & =\int \frac{d^{4} p}{(2 \pi)^{4}}|p><p| .
\end{aligned}
$$

With these definitions, we write equation (4) in the following manner:

$$
\mathcal{G}=G_{B S}-G_{B S} V \mathcal{G}
$$

and equation (5):

$$
\mathcal{G}=G_{B S}-\mathcal{G} V G_{B S} .
$$




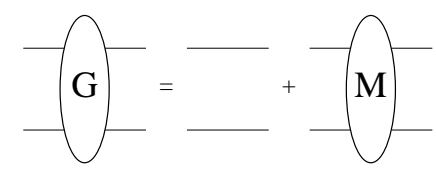

Figure 4: Scattering matrix.

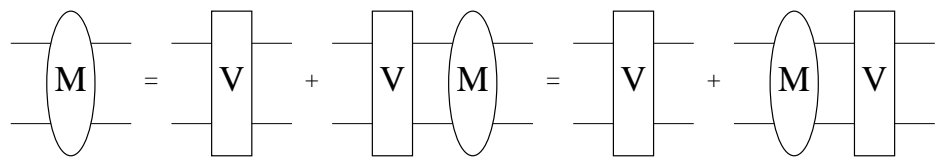

Figure 5: Bethe-Salpeter equation.

\subsection{Scattering Matrix and Unitarity}

The connected part of the four-point propagator, $\mathcal{G}$, with the external legs amputated (cf. Fig. 4) is called the scattering matrix, $\mathcal{M}$ :

$$
\mathcal{G}=G_{B S}-G_{B S} \mathcal{M} G_{B S} .
$$

The Bethe-Salpeter equation for the scattering matrix is a direct consequence of the definition of the scattering matrix given by the last equation and the basic integral equation for the four-point propagator, (11) or (12):

$$
\mathcal{M}=V-V G_{B S} \mathcal{M},
$$

or

$$
\mathcal{M}=V-\mathcal{M} G_{B S} V
$$

respectively. This pair of equations is illustrated by Fig. 5, where all external legs are actually amputated.

The Bethe-Salpeter equations for the conjugate $\mathcal{M}$ matrix (which is just complex conjugated in the scalar case) look very similar:

$$
\mathcal{M}^{\dagger}=V^{\dagger}-\mathcal{M}^{\dagger} G_{B S}^{\dagger} V^{\dagger} .
$$


or

$$
\mathcal{M}^{\dagger}=V^{\dagger}-V^{\dagger} G_{B S}^{\dagger} \mathcal{M}^{\dagger}
$$

From equation (14), we see that

$$
\mathcal{M}=V\left(1-G_{B S} \mathcal{M}\right)
$$

and from equation (16):

$$
\mathcal{M}^{\dagger}=\left(1-\mathcal{M}^{\dagger} G_{B S}^{\dagger}\right) V^{\dagger}
$$

After left multiplication of (18) by $1-\mathcal{M}^{\dagger} G_{B S}^{\dagger}$ and right multiplication of (19) by $1-G_{B S} \mathcal{M}$, the pair of equations (18) and (19) becomes

$$
\begin{aligned}
\left(1-\mathcal{M}^{\dagger} G_{B S}^{\dagger}\right) \mathcal{M} & =\left(1-\mathcal{M}^{\dagger} G_{B S}^{\dagger}\right) V\left(1-G_{B S} \mathcal{M}\right), \\
\mathcal{M}^{\dagger}\left(1-G_{B S} \mathcal{M}\right) & =\left(1-\mathcal{M}^{\dagger} G_{B S}^{\dagger}\right) V^{\dagger}\left(1-G_{B S} \mathcal{M}\right) .
\end{aligned}
$$

Subtraction of (21) from (20) results in the unitarity condition for the BetheSalpeter $\mathcal{M}$ matrix:

$$
\begin{aligned}
\mathcal{M}-\mathcal{M}^{\dagger}= & -\mathcal{M}^{\dagger}\left(G_{B S}-G_{B S}^{\dagger}\right) \mathcal{M} \\
& +\left(1-\mathcal{M}^{\dagger} G_{B S}^{\dagger}\right)\left(V-V^{\dagger}\right)\left(1-G_{B S} \mathcal{M}\right) .
\end{aligned}
$$

The positive and negative energy elastic cuts are discontinuities of the $\mathcal{M}$ matrix associated with the discontinuities of the Bethe-Salpeter propagator, $G_{B S}-G_{B S}^{\dagger}$, which are proportional to the $\delta$-functions restricting two nucleons on their mass shells. Since this can only happen when $P^{2} \geq 4 m^{2}$, we have the positive $(W \geq 2 m)$ and negative $(W \leq-2 m)$ energy cuts on the $W$ plane, where $W$ is the invariant mass of the system: $P^{2}=W^{2}$. The discontinuities of the kernel, $V-V^{\dagger}$, associated with at least one exchange boson on-shell give rise to a series of overlapping cuts with thresholds at $W= \pm(2 m+N \mu)$ with $N \geq 1$ and $\mu$ being the boson mass. 


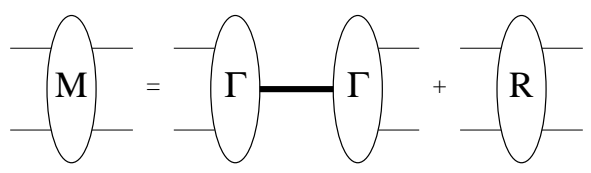

Figure 6: Bound state.

\subsection{Bound State and Wave Equation}

A bound state is associated with another type of discontinuity of the scattering matrix: if there exists a bound state for the two-body system at some particular value of the total four-momentum $P$ such that $P^{2}=M^{2}$, where $M$ is the mass of the bound state, then the scattering matrix has a pole at $P^{2}=M^{2}$. The graphical representation of the $\mathcal{M}$ matrix with the bound state pole,

$$
\mathcal{M}=\frac{|\Gamma><\Gamma|}{P^{2}-M^{2}}+\mathcal{R}
$$

is given by Fig. 6. In equation (23), $\mid \Gamma>$ is called the Bethe-Salpeter bound state vertex function and $\mathcal{R}$ is regular at $P^{2} \rightarrow M^{2}$.

If we now substitute this form of the scattering matrix (23) into the BetheSalpeter equation (14), then, equating residues and omitting $<\Gamma \mid$ on both sides, we obtain the Bethe-Salpeter wave equation for the bound state vertex function:

$$
\left(1+V G_{B S}\right) \mid \Gamma>=0,
$$

illustrated by Fig. 7 [4].

Given the definition of the Bethe-Salpeter bound state wave function,

$$
\left|\psi>=G_{B S}\right| \Gamma>
$$

we write

$$
\left(G_{B S}^{-1}+V\right) \mid \psi>=0
$$




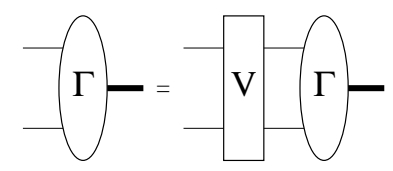

Figure 7: Bethe-Salpeter equation for the vertex function.

The last equation can have an even more elegant form if we observe the equivalence (as soon as both $G_{B S}^{-1}$ and $\mathcal{G}^{-1}$ exist) of equation (11) and this definition:

$$
\mathcal{G}^{-1}=G_{B S}^{-1}+V .
$$

The Bethe-Salpeter wave equation (26) can now be rewritten as

$$
\mathcal{G}^{-1} \mid \psi>=0 \text {. }
$$

One has to be very careful with equations like the last one. Its meaning is that the matrix elements of operator $\mathcal{G}^{-1} \mid \psi>$ in the momentum space are zero, which should be clear from the previous discussion. Indeed, the bound state wave function $|\psi\rangle$ is defined only for the total momentum $P$ such that $P^{2}=M^{2}$. At this particular value of the total momentum the fourpoint propagator has a singularity associated with the bound state pole of the scattering matrix $\mathcal{M}$. Strictly speaking, the operator $\mathcal{G}$ does not exist at $P^{2}=M^{2}$. It is natural then that the matrix elements of the product of its inverse with an operator that exists only at $P^{2}=M^{2}$ are zero.

A similar discussion can be repeated here with respect to the scattering states. Singularities associated with the bound state are clearly not the only singularities of the four-point propagator. As it was mentioned above, the singularity structure of the free two-body propagator $G_{B S}$ is responsible for the elastic cut of the scattering matrix. But the scattering states-defined 
in terms of the particle on-shell plane waves - exist only at those values of momenta where $G_{B S}$ is singular. Thus, the form of the wave equation for the scattering states is the same as (28).

The above two paragraphs are in the perfect agreement with a well-known fact from linear algebra that if a linear operator $\mathcal{O}$ has some non-trivial kernel $\operatorname{Ker}(\mathcal{O})$, then $\mathcal{O}^{-1}$ is not defined on $\operatorname{Ker}(\mathcal{O})$ [20]. In our case, $\mathcal{O}=\mathcal{G}^{-1}$.

The simpliest illustration of the kind of misleading conclusions we have to beware of is the following chain of relations:

$$
(2 \pi)^{4} \delta^{4}\left(p_{1}^{\prime}-p_{1}\right)=<p_{1}^{\prime}\left|p_{1}>=<p_{1}^{\prime}\right| \mathbf{1}\left|p_{1}>=<p_{1}^{\prime}\right| G_{1} G_{1}^{-1} \mid p_{1}>.
$$

Everything is irreproachable until the particle is on-shell. In this case, one might erroneously conclude that the last expression is zero since $G_{1}^{-1} \mid p_{1}>=0$ for $p_{1}: p_{1}^{2}=m^{2}$. From the mathematical point of view, the essence of the problem is that the operator representation

$$
\mathbf{1}=G_{1} G_{1}^{-1}
$$

is not valid at $p_{1}^{2}=m^{2}$, since operator $G_{1}^{-1}$ does not, strictly speaking, exist at this value of momentum.

Suppose, however, that, after some algebraic transformations, we observe the operator string of the form $\mathcal{O O}^{-1}$, where $\mathcal{O}$ has some singularity structure. Then the rule of the thumb we heavily use in Chapters 3 and 4 is to collapse $\mathcal{O O}^{-1}$ into 1 before considering matrix elements of the expression in question, so that we guarantee that the analytical structure of the corresponding integral equations is taken care of automatically. 


\subsection{Normalization Condition}

The normalization of the bound state vertex function can also be derived from the Bethe-Salpeter equations (14) and (15) [4]. If we substitute $V$ from (15) as $\mathcal{M}+\mathcal{M} G_{B S} V$ into (14), we obtain the nonlinear symmetric Bethe-Salpeter equation for the $\mathcal{M}$ matrix,

$$
\mathcal{M}=V-\mathcal{M} G_{B S} \mathcal{M}-\mathcal{M} G_{B S} V G_{B S} \mathcal{M}
$$

After substituting the expression for $\mathcal{M}$ in the presence of a bound state, (23), the last equation gives

$$
\begin{aligned}
\frac{|\Gamma><\Gamma|}{P^{2}-M^{2}}+\mathcal{R}= & V-\left(\frac{|\Gamma><\Gamma|}{P^{2}-M^{2}}+\mathcal{R}\right) G_{B S}\left(\mathcal{R}+\frac{|\Gamma><\Gamma|}{P^{2}-M^{2}}\right) \\
& -\left(\frac{|\Gamma><\Gamma|}{P^{2}-M^{2}}+\mathcal{R}\right) G_{B S} V G_{B S}\left(\mathcal{R}+\frac{|\Gamma><\Gamma|}{P^{2}-M^{2}}\right)
\end{aligned}
$$

which reduces to

$$
\begin{aligned}
|\Gamma><\Gamma|= & -\lim _{P^{2} \rightarrow M^{2}}\left\{\frac{\left|\Gamma>\left(<\Gamma\left|G_{B S}\right| \Gamma>+<\Gamma\left|G_{B S} V G_{B S}\right| \Gamma>\right)<\Gamma\right|}{P^{2}-M^{2}}\right. \\
& +|\Gamma><\Gamma|\left(G_{B S} \mathcal{R}+G_{B S} V G_{B S} \mathcal{R}\right) \\
& \left.+\left(\mathcal{R} G_{B S}+\mathcal{R} G_{B S} V G_{B S}\right)|\Gamma><\Gamma|\right\}
\end{aligned}
$$

after multiplication by $P^{2}-M^{2}$ and keeping only those terms that are not zero at $P^{2} \rightarrow M^{2}$. The last two lines vanish because of the wave equation (24), and what remains is

$$
\lim _{P^{2} \rightarrow M^{2}} \frac{<\Gamma\left|\left(G_{B S}+G_{B S} V G_{B S}\right)\right| \Gamma>}{P^{2}-M^{2}}=-1 .
$$

We have to expand this now about $P^{2}=M^{2}$ using L'Hopital's rule:

$$
<\Gamma\left|\left(G_{B S}^{\prime}+\left(G_{B S} V G_{B S}\right)^{\prime}\right)\right| \Gamma>=-1
$$


where

$$
G_{B S}^{\prime}=\frac{\partial G_{B S}}{\partial P^{2}}=2 P^{\mu} \frac{\partial G_{B S}}{\partial P_{\mu}}
$$

and similarly for $\left(G_{B S} V G_{B S}\right)^{\prime}$, and where we omitted all terms involving derivatives of the vertex function since they are zero because of the wave equation.

Expression (35) can also be further simplified. Using the Bethe-Salpeter wave equation to obtain

$$
<\Gamma\left|\left(G_{B S} V G_{B S}\right)^{\prime}\right| \Gamma>=<\Gamma\left|G_{B S} V^{\prime} G_{B S}\right| \Gamma>-2<\Gamma\left|G_{B S}^{\prime}\right| \Gamma>
$$

and noticing that, due to the standard differentiation rules,

$$
-<\Gamma\left|G_{B S}^{\prime}\right| \Gamma>=<\Gamma\left|G_{B S}\left(G_{B S}^{-1}\right)^{\prime} G_{B S}\right| \Gamma>,
$$

we finally write the normalization of the bound state vertex function in the following manner:

$$
<\psi\left|\left(\mathcal{G}^{-1}\right)^{\prime}\right| \psi>=-1 .
$$

\subsection{Current Operator and Five-point Func- tion}

The electromagnetic properties of bound and scattering states are described by the matrix elements $\mathcal{J}^{\mu}$ of the effective current operator $J^{\mu}$ :

$$
\mathcal{J}^{\mu}=<\psi\left|J^{\mu}\right| \psi>
$$

The structure of the current $J^{\mu}$ can be obtained by attaching a virtual photon leg to all lines of the corresponding Feynman diagram. Fig. 8 gives a graphical 


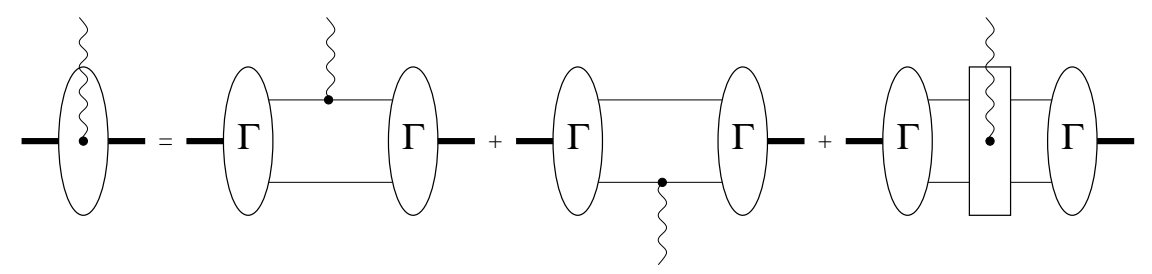

Figure 8: Current operator.

representation of this scenario. The last term in Fig. 8 sums contributions from the photon interaction with all members of Fig. 2, where we again imply that a photon leg is successively attached to all lines (if both the bound state constituents and interaction bosons carry the charge).

The last term on Fig. 8 is called the exchange current, $J_{\mathrm{ex}}^{\mu}$, and the algebraic expression for $J^{\mu}$ can be then written as:

$$
J^{\mu}=J_{\text {IA }}^{\mu}+J_{\text {ex }}^{\mu}
$$

where

$$
J_{\text {IA }}^{\mu}=i J_{1}^{\mu} G_{2}^{-1}+i J_{2}^{\mu} G_{1}^{-1}
$$

and $J_{i}^{\mu} ; i=1,2$; are the one-body currents. The inverse one-body propagators appear to cancel the extraneous one-body propagators coming from the definition of the bound state wave function (25).

The five-point function, $\mathcal{G}^{\mu}$, another object we will need, can be also defined using $J^{\mu}$ :

$$
\mathcal{G}^{\mu}=-\mathcal{G} J^{\mu} \mathcal{G}
$$

Here we see again (cf. Fig. 9) that inverse propagators in the definition of $J_{\text {IA }}^{\mu}$ are canceled with extra propagators from $\mathcal{G}$. It can be shown [14] that 


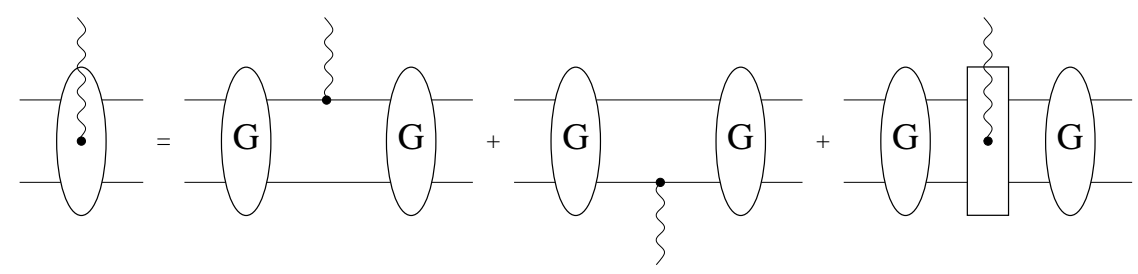

Figure 9: Five-point function.

definition (43) is equivalent to the conventional definition of the five-point function.

The scattering operator $\mathcal{C}^{\mu}$ is defined as the five-point function represented on Fig. 9 with all external legs amputated,

$$
\mathcal{G}^{\mu}=-G_{B S} \mathcal{C}^{\mu} G_{B S}
$$

This definition is analogous to the definition of the scattering matrix $\mathcal{M}(13)$ in the sense that the scattering operator is related to the five-point function in the same way as the scattering matrix is related to the four-point function. And even more important observation is that both the scattering matrix and the connected part of the scattering operator are directly related to a physical observable (the differential cross-section).

The conservation of the current $J^{\mu}[14,21]$ follows from the one- and twobody Ward-Takahashi identities for the one-body [13] and exchange [14, 21] currents respectively.

A regular form of the one-body Ward-Takahashi identities,

$$
q_{\mu} J_{i}^{\mu}\left(p_{i}+q, p_{i}\right)=-e_{i}\left(G_{i}^{-1}\left(p_{i}+q\right)-G_{i}^{-1}\left(p_{i}\right)\right),
$$

can be replaced by their operator form,

$$
q_{\mu} J_{i}^{\mu}=\left[\hat{e}_{i}(q), G_{i}^{-1}\right]
$$


if we introduce the charge operators $\hat{e}_{i}(q)$ by their matrix elements in the momentum space:

$$
<p_{i}^{\prime}\left|\hat{e}_{i}(q)\right| p_{i}>=(2 \pi)^{4} \delta^{4}\left(p_{i}^{\prime}-p_{i}-q\right) e_{i}
$$

where $e_{i}$ is the isospin part of the charge operator of the $i$ th particle, $\frac{1}{2}\left(1+\tau_{3}^{(i)}\right)$. So defined charge operators, $\hat{e}_{i}$, act as the momentum shift operators in the momentum space and as the usual charge operators in the isospin space. From now on, we will use the same notation, $e_{i}$, for the charge operators in the momentum space as well.

With these definitions, a well-known result [14, 21] for the exchange current divergence,

$$
\begin{aligned}
q_{\mu} J_{\mathrm{ex}}^{\mu}= & e_{1} V\left(p^{\prime}-\frac{q}{2}, p ; P\right)+e_{2} V\left(p^{\prime}+\frac{q}{2}, p ; P\right) \\
& -V\left(p^{\prime}, p+\frac{q}{2} ; P^{\prime}\right) e_{1}-V\left(p^{\prime}, p-\frac{q}{2} ; P^{\prime}\right) e_{2},
\end{aligned}
$$

reduces to

$$
q_{\mu} J_{\mathrm{ex}}^{\mu}=\left[e_{\Sigma}, V\right]
$$

where we used

$$
e_{\Sigma}=e_{1}+e_{2}
$$

The proof of the current conservation is a one-line task now. The divergence of the current $J^{\mu}$,

$$
q_{\mu} J^{\mu}=i\left[e_{1}, G_{1}^{-1}\right] G_{2}^{-1}+i\left[e_{2}, G_{2}^{-1}\right] G_{1}^{-1}+\left[e_{\Sigma}, V\right]=\left[e_{\Sigma}, \mathcal{G}^{-1}\right]
$$

along with the wave equation (28) yields

$$
q_{\mu} \mathcal{J}^{\mu}=0
$$




\subsection{Charge Conservation}

The last observation we make in this chapter is about the equivalence of the normalization condition (39) for the bound state consisting of two particles of the same isospin and the charge conservation [4, 22]. For this end, we have to consider the one-body Ward-Takahashi identities (45) in the $q \rightarrow 0$ limit:

$$
q_{\mu} J_{i}^{\mu}=\left[e_{i}(q), G_{i}^{-1}\right] \stackrel{q \rightarrow 0}{\longrightarrow}-e_{i} q_{\mu} \frac{\partial}{\partial p_{\mu}^{(i)}} G_{i}^{-1},
$$

or, to be more general, given the definition of the charge operator (47), we can conclude that for any operator in the momentum space of the $i$ th particle

$$
\left[e_{i}(q), \hat{\mathcal{O}}\right] \stackrel{q \rightarrow 0}{\longrightarrow}-e_{i} q_{\mu} \frac{\partial}{\partial p_{\mu}^{(i)}} \hat{\mathcal{O}}
$$

This allows us to write

$$
q_{\mu} J_{\mathrm{IA}}^{\mu} \stackrel{q \rightarrow 0}{\longrightarrow}-q_{\mu}\left(e_{1} \frac{\partial}{\partial p_{\mu}^{(1)}}+e_{2} \frac{\partial}{\partial p_{\mu}^{(2)}}\right) G_{B S}^{-1} .
$$

Since

$$
\begin{aligned}
\frac{\partial}{\partial p_{\mu}^{(1)}} & =\frac{\partial}{\partial P_{\mu}}+\frac{1}{2} \frac{\partial}{\partial p_{\mu}} \\
\frac{\partial}{\partial p_{\mu}^{(2)}} & =\frac{\partial}{\partial P_{\mu}}-\frac{1}{2} \frac{\partial}{\partial p_{\mu}}
\end{aligned}
$$

expression (55) transforms to

$$
q_{\mu} J_{\mathrm{IA}}^{\mu} \stackrel{q \rightarrow 0}{\longrightarrow}-q_{\mu}\left(e_{\Sigma} \frac{\partial}{\partial P_{\mu}}+\frac{1}{2}\left(e_{1}-e_{2}\right) \frac{\partial}{\partial p_{\mu}}\right) G_{B S}^{-1} .
$$

The easiest way to find the zero momentum transfer limit for the exchange current divergence is to directly consider the expansion of the two-body WardTakahashi identity (48), which gives

$$
q_{\mu} J_{\mathrm{ex}}^{\mu} \stackrel{q \rightarrow 0}{\longrightarrow}\left[e_{\Sigma}, V\right]_{\tau}-q_{\mu}\left\{\frac{\partial V}{\partial P_{\mu}} e_{\Sigma}+\frac{e_{1}-e_{2}}{2} \frac{\partial V}{\partial p_{\mu}^{\prime}}+\frac{\partial V}{\partial p_{\mu}} \frac{e_{1}-e_{2}}{2}\right\},
$$


where by $[,]_{\mathcal{\tau}}$ we denoted the commutator in the isospin space. A natural assumption is that the first term in the last expression is zero. It is obviously true for the following form of the kernel

$$
V=V_{0}+\vec{\tau}^{(1)} \cdot \vec{\tau}^{(2)} V_{\tau}
$$

since $\vec{\tau}^{(1)} \cdot \vec{\tau}^{(2)}$ commutes with $\tau_{3}^{(1)}+\tau_{3}^{(2)}$.

With this assumption expression (59) becomes

$$
q_{\mu} J_{\mathrm{ex}}^{\mu} \stackrel{q \rightarrow 0}{\longrightarrow}-q_{\mu}\left\{e_{\Sigma} \frac{\partial V}{\partial P_{\mu}}+\frac{e_{1}-e_{2}}{2} \frac{\partial V}{\partial p_{\mu}^{\prime}}+\frac{\partial V}{\partial p_{\mu}} \frac{e_{1}-e_{2}}{2}\right\} .
$$

We are considering particles of the same isospin. In this case $e_{1}=e_{2}=\frac{1}{2}\left(1+\tau_{3}\right)$, and collecting results from expressions (58) and (61), we write

$$
q_{\mu} J^{\mu} \stackrel{q \rightarrow 0}{\longrightarrow}-q_{\mu} e_{\Sigma} \frac{\partial}{\partial P_{\mu}} \mathcal{G}^{-1}
$$

This limit uniquely determines the longitudinal part of the current operator $J^{\mu}$ at the zero momentum transfer:

$$
J^{\mu}(0)=-e_{\Sigma} \frac{\partial}{\partial P_{\mu}} \mathcal{G}^{-1}=-2 P^{\mu} e_{\Sigma}\left(\mathcal{G}^{-1}\right)^{\prime} .
$$

Given this form of the current at the zero limit along with the normalization condition (39), we conclude that

$$
\mathcal{J}^{\mu}(0)=2 P^{\mu} e_{\Sigma},
$$

which is a transparent indication that the charge is conserved. 


\section{Chapter 3}

\section{Quasipotential Formalism}

\subsection{Motivations and General Requirements}

By no means is the Bethe-Salpeter equation the only possible form of relativistic equation for the scattering matrix $\mathcal{M}$. A simple observation is that equation (14) is equivalent to the pair of equations

$$
\begin{aligned}
\mathcal{M} & =U-U g \mathcal{M}, \\
U & =V-V \Delta G U,
\end{aligned}
$$

where $g$ and $U$ are called the quasipotential two-body propagator and the quasipotential kernel respectively and where

$$
\Delta G=G_{B S}-g .
$$

Indeed, the following chain of relations demonstrates this equivalence.

$$
\begin{aligned}
\mathcal{M} & =U-U g \mathcal{M} \\
& =(V-V \Delta G U)-(V-V \Delta G U) g \mathcal{M}
\end{aligned}
$$




$$
\begin{aligned}
& =V-V \Delta G U-V g \mathcal{M}+V \Delta G U g \mathcal{M} \\
& =V-V g \mathcal{M}-V \Delta G(U-U g \mathcal{M}) \\
& =V-V g \mathcal{M}-V \Delta G \mathcal{M} \\
& =V-V G_{B S} \mathcal{M}
\end{aligned}
$$

Thus, we are faced with the whole spectrum of relativistic two-body equations. All of them (except some particular cases mentioned at the end of this section) can be thought as being generated by the initial choice of the quasipotential propagator $g$. The motivations for choosing any specific form of the propagator and the minimal requirements on it will be discussed in a moment. As soon as a propagator is chosen, it uniquely determines the quasipotential kernel $U$ by means of equation (66). It is clear that this kernel no longer represents a sum of Feynman diagrams with the standard rules. Some additional rules can be introduced to view the quasipotential kernel as a resummation of pieces of the Bethe-Salpeter kernel truncated at different numbers of boson exchanges (this is discussed in more detail in Section 5.1). Given $U$, the equation we have to solve then is (65).

\subsubsection{Motivations and Requirements}

It might seem confusing that, instead of one "simple" equation (14), we now have two. However, since the kernel $V$ includes contributions from an infinite series of the two-body irreducible Feynman diagrams, it is often truncated at some number of boson exchanges (the exact solution for the Bethe-Salpeter equation using the path integral representation [5] and requiring a significant amount of the computer time was demonstrated for the scalar case only). The usual technique is to truncate the kernel at the one boson exchange (OBE) 
level (ladder approximation).

Even with these approximations, the Bethe-Salpeter equation, as any fourdimensional integral equation, is still difficult to solve numerically. It can be reduced to the two-dimensional integral equation after partial wave expansion in the center of mass frame. The remaining integrations are with respect to the relative energy and the magnitude of the relative 3-momentum. The number of operations necessary to numerically solve a two-dimensional integral equation is of the order of $N^{6}$, where $N$ is a number of point in the grid, on which the integral is approximated by a sum. Comparison of $N^{6}$ with $N^{3}$, a number of operations solving one-dimensional integral equations, is rather self-explaining. This is a clear indication of why three-dimensional reductions of the Bethe-Salpeter equations are of such importance.

Therefore, a common feature of all considered quasipotential propagators is that they constrain the relative four-momentum in some way reducing the phase space of the system to some submanifold of the total phase space available in the Bethe-Salpeter case, whereas contributions coming from the rest of the phase space are incorporated into the new kernel $U$ by means of (66).

The central idea of the method is to choose the quasipotential propagator in such a way that operator $\Delta G$ could be considered small in some sense. If it is small enough, then we can truncate series generated by equation (66) at the 0th order approximating the quasipotential kernel $U$ by the Bethe-Salpeter kernel $V$. Since the relative momentum is constrained (by explicit presence of the $\delta$-function, for example), instead of tackling the four-dimensional integral equation, we have to solve the three-dimensional equation (65), which can be reduced to one-dimensional by the procedure described above.

Except for this desirable feature of generating three-dimensional reduction, 
the only other strict requirement we impose upon a quasipotential propagator is that it must preserve the positive energy elastic cut (22) at any order of truncation. Starting with the quasipotential equation for the $\mathcal{M}$ matrix (65) and proceeding similarly to how we obtained the unitarity condition (22), we end up with:

$$
\begin{aligned}
\mathcal{M}-\mathcal{M}^{\dagger}= & -\mathcal{M}^{\dagger}\left(g-g^{\dagger}\right) \mathcal{M} \\
& +\left(1-\mathcal{M}^{\dagger} g^{\dagger}\right)\left(U-U^{\dagger}\right)(1-g \mathcal{M}) .
\end{aligned}
$$

Thus, the elastic cut requirement for the quasipotential propagator can be formulated as:

$$
\begin{aligned}
g-g^{\dagger} \stackrel{.}{=} & G_{B S}-G_{B S}^{\dagger}=2 \pi i \delta\left(\left(\frac{1}{2} P+p\right)^{2}-m^{2}\right) \delta\left(\left(\frac{1}{2} P-p\right)^{2}-m^{2}\right) \\
& . . \\
&
\end{aligned}
$$

where under "..”" sign we understand an equation that is necessarily held only on the mass shell $W=2 E_{p}$, where $E_{p}=\sqrt{\mathbf{p}^{2}+m^{2}}$ is the on-shell energy. The last line in (70) is evaluated in the center of mass frame.

In other words, the choice of a quasipotential propagator must preserve physics along the positive energy elastic cut at any order and can describe it differently in non-physical regions. (However, the exact solution for the $\mathcal{M}$ matrix will always be the same, as was mentioned at the beginning of this section.)

The above requirements leave a number of degrees of freedom in choosing $g$. And it is not surprising then that a variety of quasipotential propagators have been proposed and considered in the literature. The optimal choice of the propagator heavily depends on the model considered. The number of boson exchanges included in the Bethe-Salpeter kernel, the level of truncation of 
the quasipotential equation (66), the masses of the bosons and nucleons, the coupling constants, and the range of kinematic variables one is interested inall play a role in producing a spectrum of motivations for selecting one or another propagator for a model.

And yet another important observation should be made at this point. Since we know the exact solution of the full Bethe-Salpeter equation (in the scalar case) [5], we can compare this exact solution with what various quasipotential approaches provide. The 0th order approximation to the quasipotential (66) implies that $U=V$. What we have to analyse next is the $V g V$ term in the iterations of (65). Expanding this term as a series of $\mu / m$, where $\mu$ is the boson mass, and comparing corresponding coefficients, one finds that all quasipotential equations turn out to possess better convergence properties than the ladder approximation to the Bethe-Salpeter equation [1]. The same picture of better convergence properties arises for the calculations of the ground state mass for the exact Bethe-Salpeter and various quasipotential equations [5]. Therefore, the issue of better convergence (along with the previously mentioned reduction property), is another reason to study quasipotential equations.

\subsubsection{Review of Proposed Quasipotential Propagators}

Two basic families of proposed quasipotential propagators (written down in the center of mass frame where $P=(W, \mathbf{0}))$ are

$$
\begin{aligned}
g(p ; W) & =\frac{2 \pi}{E_{p}} \delta\left(p_{0}\right) \frac{f\left(4 E_{p}^{2}, W^{2}\right)}{4 E_{p}^{2}-W^{2}-i \epsilon} \\
g(p ; W) & =\frac{2 \pi}{E_{p}} \delta\left(p_{0}-\left(E_{p}-W / 2\right)\right) \frac{f\left(4 E_{p}^{2}, W^{2}\right)}{4 E_{p}^{2}-W^{2}-i \epsilon}
\end{aligned}
$$


where $f$ is an arbitrary function of two arguments and it is equal to 1 on its diagonal: $f(x, x)=1$. This condition guarantees that the elastic cut requirement (70) is satisfied. Both families have their explicitly relativistic representations:

$$
\begin{aligned}
g(p ; P)= & 2 \pi \int_{4 m^{2}}^{\infty} \frac{d s^{2}}{s^{2}-W^{2}-i \epsilon} f\left(s^{2}, W^{2}\right) \\
& \times \delta^{(+)}\left(\left(\frac{s}{2 W} P+p\right)^{2}-m^{2}\right) \delta^{(+)}\left(\left(\frac{s}{2 W} P-p\right)^{2}-m^{2}\right), \\
g(p ; P)= & 2 \pi \int_{4 m^{2}}^{\infty} \frac{d s^{2}}{s^{2}-W^{2}-i \epsilon} f\left(s^{2}, W^{2}\right) \\
& \times \delta^{(+)}\left(\left(\frac{1}{2} P+p\right)^{2}-m^{2}\right) \delta^{(+)}\left(\left(\frac{2 s-W}{2 W} P-p\right)^{2}-m^{2}\right),
\end{aligned}
$$

where $\delta^{(+)}$indicated that only the positive energy root of the $\delta$-function must be included.

The major difference between these two approaches is that in the first case both particles are off-shell and only three-momentum is transferred, while in the second case one particle is on-shell in initial, final and all intermediate states. The latter case was designed to work for systems consisting of one heavy and one light particles, when the natural idea is to place the heavy particle onshell. Since the on-shell family does not treat particles symmetrically (like the former case does), some explicit symmetrization procedures [23] are required when considering equal mass two-particle systems.

Different choices of function $f$ for both cases generate different propagators $[1,24]$. Table 1 summarizes some of the possible choices along with the authors who proposed them.

At this point one might erroneously conclude that $p_{0}=0$ and $p_{0}=E_{p}-W / 2$ are the only two possible submanifolds to which the available phase space can be reduced by a quasipotential propagator. This is obviously 
Table 1: Proposed families of quasipotential propagators.

\begin{tabular}{|c|c|c|}
\hline$f\left(x^{2}, y^{2}\right)$ & $\delta\left(p_{0}\right)$ & $\delta\left(p_{0}-\left(E_{p}-W / 2\right)\right)$ \\
\hline \hline 1 & Blankenbecler-Sugar [25] & Erkelenz-Holinde [26] \\
\hline$\frac{x}{y}$ & Todorov [27] & - \\
\hline$\frac{x+y}{2 x}$ & - & Kadychevsky [28] \\
\hline$\frac{x+y}{2 y}$ & Thompson [29] & Gross [30] \\
\hline
\end{tabular}

not true. To demonstrate this, we introduce a new superfamily of propagators similar to that one suggested by Gross [31]. Modifying the argument of the $\delta$-functions in (73) or (74), we can write, for example, the following:

$$
\begin{aligned}
g(p, P ; \nu)= & 2 \pi \int_{4 m^{2}}^{\infty} \frac{d s^{2}}{s^{2}-W^{2}-i \epsilon} f\left(s^{2}, W^{2} ; \nu\right) \\
& \times \delta^{(+)}\left(\left(\frac{(1-\nu) s+\nu W}{2 W} P+p\right)^{2}-m^{2}\right) \\
& \times \delta^{(+)}\left(\left(\frac{(1+\nu) s-\nu W}{2 W} P-p\right)^{2}-m^{2}\right),
\end{aligned}
$$

where $\nu$ is a real number such that $-1 \leq \nu \leq 1$, and $f(x, y ; \nu)$ is a function equal to 1 on its diagonal, $f(x, x ; \nu)=1$, for any value of parameter $\nu$ to guarantee that the positive energy elastic cut requirement (70) is satisfied. A particular choice of function $f$,

$$
f(x, y ; \nu)=\frac{x}{y} \cdot \frac{x+y}{\left(1+\nu^{2}\right) x+\left(1-\nu^{2}\right) y}
$$

produces the series of quasipotential propagators considered by Gross in [31].

Representation (75) can be integrated in the center of mass frame to yield

$$
g(p, W ; \nu)=\frac{2 \pi}{E_{p}} \delta\left(p_{0}-\nu\left(E_{p}-W / 2\right)\right) \frac{f\left(4 E_{p}^{2}, W^{2} ; \nu\right)}{4 E_{p}^{2}-W^{2}-i \epsilon} .
$$


It is clear now that if $\nu=1(-1)$, then the first (second) particle is on-shell, and if $\nu=0$, then the both particle is off-shell and the 3-momentum only is transferred. If function $f$ has the form as in (76), then for $\nu=1$ we obtain the original Gross propagator and for $\nu=0$ we end up with Todorov's case (cf. Table 1). However, the main point of this family of propagators is that the whole spectrum $p_{0}=\nu\left(E_{p}-W / 2\right)$ of submanifolds is permitted.

And again, we are quite far from exhausting all possible three-dimensional reductions. Klein and Lee [32] considered a case such that the reduction procedure could not be made equivalent to just a quasipotential propagator substitution, and an explicit projection of the scattering matrix and the kernel onto the considered subspace of the phase space is required. Phillips and Wallace followed the ideas of Klein and Lee and generalized yet another quasipotential reduction model developed by one of the authors in [33, 34]. The result was an even more innovative approach (the equal-time equation) [17] not incorporating the explicit presence of $\delta$-functions. However, the inevitable common property of all possible three-dimensional reductions is that the phase space is reduced to some submanifold first, then the scattering matrix is projected onto it and a new quasipotential propagator is afterwards introduced in one way or another (in the simpliest case considered by Phillips and Wallace- - by means of integrating the relative energy dependence out of the free two-body Bethe-Salpeter propagator,

$$
g=\int \frac{d p_{0}}{2 \pi} G_{B S},
$$

which is equivalent to ignoring the time dependence of the propagator. A covariant generalization of this approach was also considered in $[35,36]$.) 
Having set off the essential characteristics of any three-dimensional reduction, we can now introduce a formal generic description of the method.

\subsection{Generic Approach}

In this section we work out a generic method of dealing with the different quasipotential approaches described in the previous section. This method is general and does not depend on the specific properties of any particular quasipotential. All results are independent of the analytical structure of the emerging integral equations generated by a particular choice of the quasipotential, and in the next chapter we demonstrate how our generic results produce the right answers for two well-known cases.

\subsubsection{Four-point Propagator}

The basic ideas of this section are rather straightforward and can be thought as a logical continuation of the fundamental relativistic quantum field theory results given in Chapter 2, where equation (13) is actually a definition of the scattering matrix. In that equation, both the four-point and the free two-body propagators, defined as the Fourier transforms of the vacuum expectation values of the time ordered products of the corresponding nucleon field operators, are not directly related to any physical observables. But the $\mathcal{M}$ matrix is (its absolute square with all external particles on-shell is proportional to the differential cross section: $\left.|\mathcal{M}|^{2} \sim \frac{d \sigma}{d \Omega}\right)$. That is why a requirement that all quasipotential propagators preserve the scattering matrix elastic cut is fundamental. However, as was mentioned in the previous section, the exact value of the scattering matrix at any point of the phase space is always the same and 


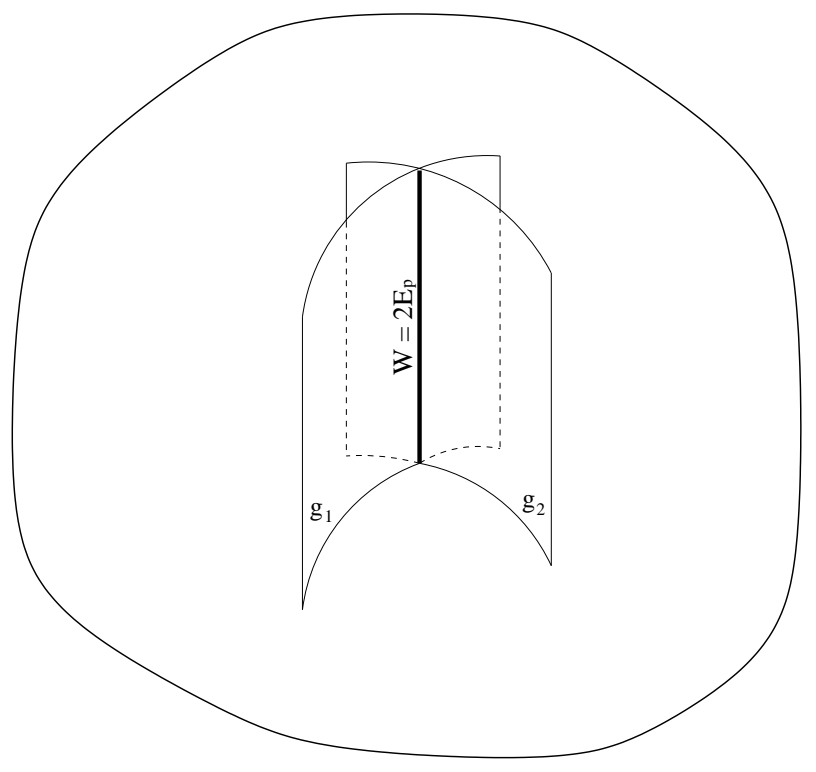

Figure 10: Phase space reduction.

it is independent of the propagator selection. These considerations allow us to write

$$
G_{B S}^{-1}-G_{B S}^{-1} \mathcal{G} G_{B S}^{-1}=\mathcal{M} \doteq g^{-1}-g^{-1} \mathcal{G}_{Q} g^{-1}
$$

This equation should be thought as a definition of the quasipotential fourpoint propagator $\mathcal{G}_{Q}$. It is a direct consequence of the scattering matrix definition given by (13). The quasipotential propagator $g$ reduces the total phase space to some submanifold. Clearly, its inverse exists only on this submanifold. The quasipotential four-point propagator can be also defined only on this submanifold. Sign "”" indicates that we are considering this equation on a reduced phase space only.

To clarify the issue completely, we consider two quasipotential propagators, $g_{1}$ and $g_{2}$ (corresponding, for example, to two different choices of parameter $\nu$ 
in the superfamily of the quasipotential propagators (75)). This leads to the two distinct submanifolds of the total phase space of the system (cf. Fig. 10). However, because of the positive energy elastic cut requirement, these two submanifolds overlap over the physical values of momenta as is shown on Fig. 10. Therefore, we can write either:

$$
g_{1}^{-1}-g_{1}^{-1} \mathcal{G}_{Q_{1}} g_{1}^{-1} \ddot{=} \mathcal{M} \ddot{=} g_{2}^{-1}-g_{2}^{-1} \mathcal{G}_{Q_{2}} g_{2}^{-1},
$$

where $\ddot{=}$ has the same meaning as in $(70)$, or:

$$
\left\{\begin{array}{l}
\mathcal{M} \doteq_{g_{1}} g_{1}^{-1}-g_{1}^{-1} \mathcal{G}_{Q_{1}} g_{1}^{-1} \\
\mathcal{M} \dot{ }_{g_{2}} g_{2}^{-1}-g_{2}^{-1} \mathcal{G}_{Q_{2}} g_{2}^{-1},
\end{array}\right.
$$

where the first (second) equation in the system of two is considered on the phase space reduced by $g_{1}\left(g_{2}\right)$.

It would be natural to expect the form of the integral equation for the quasipotential four-point function to be analogous to (11) or (12). Indeed,

$$
\begin{aligned}
\mathcal{G}_{Q} & \doteq g-g \mathcal{M} g \\
& \doteq g-g(U-U g \mathcal{M}) g \doteq g-g U(g-g \mathcal{M} g) \doteq g-g U \mathcal{G}_{Q} \\
& \doteq g-g(U-\mathcal{M} g U) g \doteq g-(g-g \mathcal{M} g) U g \doteq g-\mathcal{G}_{Q} U g
\end{aligned}
$$

Using (82), we write

$$
\begin{aligned}
\left(g^{-1}+U\right) \mathcal{G}_{Q} & \doteq\left(g^{-1}+U\right)\left(g-g U \mathcal{G}_{Q}\right) \doteq 1-U \mathcal{G}_{Q}+U g-U g U \mathcal{G}_{Q} \\
& \doteq 1-U g+U g U \mathcal{G}_{Q}+U g-U g U \mathcal{G}_{Q} \doteq 1
\end{aligned}
$$

or, with the help of (83),

$$
\begin{aligned}
\mathcal{G}_{Q}\left(g^{-1}+U\right) & \doteq\left(g-\mathcal{G}_{Q} U g\right)\left(g^{-1}+U\right) \doteq 1-\mathcal{G}_{Q} U+g U-\mathcal{G}_{Q} U g U \\
& \doteq 1-g U+\mathcal{G}_{Q} U g U+g U-\mathcal{G}_{Q} U g U \doteq 1
\end{aligned}
$$


This implies that the expression for the inverse quasipotential four-point propagator is similar to $(27)$ :

$$
\mathcal{G}_{Q}^{-1} \doteq g^{-1}+U .
$$

The quasipotential equation for the scattering matrix (65) is valid at any point of the phase space, hence, at any point of the reduced phase space as well:

$$
\mathcal{M} \doteq U-U g \mathcal{M}
$$

or

$$
\mathcal{M} \doteq U-\mathcal{M} g U
$$

Substitution of the form of the $\mathcal{M}$ matrix in the presence of the bound state (23) into any of these expressions and equating the residues with afterwards omitting of $<\Gamma \mid$ or $\mid \Gamma>$ on both sides give

$$
|\Gamma>\doteq-U g| \Gamma>
$$

or

$$
<\Gamma|\doteq-<\Gamma| g U .
$$

A natural definition of the quasipotential bound state wave function

$$
\left|\psi_{Q}>\doteq g\right| \Gamma>
$$

reduces (89) and (90) to the following form of the wave equation:

$$
<\psi_{Q}\left|\mathcal{G}_{Q}^{-1} \doteq \mathcal{G}_{Q}^{-1}\right| \psi_{Q}>\doteq 0
$$

analogous to (28). It is important to note that the last representation of the wave equation is valid only on the already reduced phase space, while both 
(89) and (90) make sense everywhere:

$$
\begin{aligned}
& |\Gamma>=-U g| \Gamma> \\
& <\Gamma|=-<\Gamma| g U .
\end{aligned}
$$

\subsubsection{Current Operator and Five-point Function}

We do not want the electromagnetic properties of the system to depend on a particular (quasipotential) approach. To be more specific, we do not expect the matrix elements (40) of the current operator to change:

$$
\mathcal{J}^{\mu}=<\psi_{Q}\left|J_{Q}^{\mu}\right| \psi_{Q}>
$$

where the quasipotential effective current $J_{Q}^{\mu}$ is to be found. Obviously, the last equation could be considered as a definition of the effective current and it could be found based on this definition. However, to expose the virtual equivalence of any quasipotential approach to the Bethe-Salpeter approach and to further demonstrate that the underlying fabric of both methods is essentially the same, we follow more rigorous and field theoretical way.

The discussion of the $\mathcal{M}$ matrix properties could be repeated here with respect to the scattering operator $\mathcal{C}^{\mu}(44)$. The result is the natural definition of the quasipotential five-point function,

$$
\mathcal{G}_{Q}^{\mu} \doteq-g \mathcal{C}^{\mu} g
$$

which is an analogue of (44). This definition along with the original definition of the scattering operator (44) gives rise to the pair of relations similar to (79),

$$
-G_{B S}^{-1} \mathcal{G}^{\mu} G_{B S}^{-1}=\mathcal{C}^{\mu} \doteq-g^{-1} \mathcal{G}_{Q}^{\mu} g^{-1}
$$


Given the quasipotential four- and five-point functions, we have all necessary machinery to construct the quasipotential effective current $J_{Q}^{\mu}$ (cf. (43)),

$$
\mathcal{G}_{Q}^{\mu} \doteq-\mathcal{G}_{Q} J_{Q}^{\mu} \mathcal{G}_{Q} .
$$

It might not seem completely clear at this point that the so-defined effective current has the same matrix elements as the Bethe-Salpeter effective current $J^{\mu}$. However, it is so due to construction. Namely, all the preceding definitions of "auxiliary" objects $\left(\mathcal{G}_{Q}, \mathcal{G}_{Q}^{\mu}\right)$ were based on the fact that the physics $\left(\mathcal{M}, \mathcal{C}^{\mu}\right)$ does not depend on one or another approach. That is why it would be natural to expect that such physical objects as the current matrix elements remain intact.

The definitions of the quasipotential four- and five-point functions were useful since they allowed us to introduce a formalism, such that all basic equations look formally the same as in the Bethe-Salpeter case, or, to be more precise, as soon as we consider dynamics on a reduced phase space, this dynamics, described in terms of the objects, analogous to those in the BetheSalpeter case, is essentially the same, no matter what particular choice of the quasipotential propagator has been made.

It must be emphasized here that this result is rather formal and it can hardly be considered as a deep insight into the field theory. The Bethe-Salpeter approach is the only valid field theoretical method from the point of view of the formalism described here. Both the quasipotential four- and five-point functions are not the Fourier transforms of the vacuum expectation values of the time ordered products of the nucleon operator fields (as they are in the Bethe-Salpeter case), but they are rather related to those by means of equations (79) and (97) respectively. The value of this formalism is that it 
reveals the simplicity of a generic quasipotential approach and allows one to write down basic definitions in a way similar to the Bethe-Salpeter case. One of the most important formulae here is the definition for a generic quasipotential current $J_{Q}^{\mu}$. It should be clear now that its matrix elements are the same as of the Bethe-Salpeter current $J^{\mu}$. However, we demonstrate this explicitly.

\subsubsection{Current Conservation}

We check first that the current is conserved (on a reduced phase space, obviously). To this end, we rewrite its definition (98) as

$$
J_{Q}^{\mu} \doteq-\mathcal{G}_{Q}^{-1} \mathcal{G}_{Q}^{\mu} \mathcal{G}_{Q}^{-1} .
$$

The ultimate purpose now is to get rid of any quasipotential $n$-point functions and to express the right hand side of this definition in terms of the BetheSalpeter effective current $J^{\mu}$, the quasipotential kernel $U$ and the free-two body propagators $G_{B S}$ and $g$. The definition of the quasipotential five-point function (97) can be used to give

$$
J_{Q}^{\mu} \doteq-\mathcal{G}_{Q}^{-1} g G_{B S}^{-1} \mathcal{G}^{\mu} G_{B S}^{-1} g \mathcal{G}_{Q}^{-1} .
$$

Substitution of the five-point function from (43) into the last expression yields

$$
J_{Q}^{\mu} \doteq \mathcal{G}_{Q}^{-1} g G_{B S}^{-1} \mathcal{G} J^{\mu} \mathcal{G} G_{B S}^{-1} g \mathcal{G}_{Q}^{-1} .
$$

Now we have $J^{\mu}$ surrounded by the strings of operators with some non-trivial analytical structure. Namely, $G_{B S}^{-1}$ has zeros where $\mathcal{G}$ has singularities and vice versa with respect to $g$ and $\mathcal{G}_{Q}^{-1}$ respectively. Following our prescription given in the previous section, we have to resolve these singularities first to guarantee that the analytical structure of this expression is taken care of adequately. 
Considering the right operator string of $J^{\mu}$ first, we write

$$
\begin{aligned}
\ldots \mathcal{G} G_{B S}^{-1} g \mathcal{G}_{Q}^{-1} & =\ldots\left(G_{B S}-G_{B S} \mathcal{M} G_{B S}\right) G_{B S}^{-1} g \mathcal{G}_{Q}^{-1} \\
& =\ldots\left(1-G_{B S} \mathcal{M}\right) g \mathcal{G}_{Q}^{-1}
\end{aligned}
$$

where we have used the scattering matrix definition (13) to cancel the singularity associated with the four-point propagator. We also moved symbol "." to its right to indicate that we are not keeping track of the constraint condition on the left side of the operator string (since it is to be left-multiplied by some other operators).

The uncertainty associated with the singularity of $g$ still remains. We deal with it as follows:

$$
\begin{aligned}
\ldots\left(1-G_{B S} \mathcal{M}\right) g \mathcal{G}_{Q}^{-1} & ={ }^{\cdot} \quad \ldots\left(1-G_{B S} \mathcal{M}\right) g\left(g^{-1}+U\right) \\
& =\cdot \ldots\left(1-G_{B S} \mathcal{M}\right)(1+g U) \\
& =\cdot \ldots\left(1+g U-G_{B S}(\mathcal{M}+\mathcal{M} g U)\right) \\
& ={ }^{\cdot} \quad \ldots\left(1+g U-G_{B S} U\right) \\
& =\ldots(1-\Delta G U),
\end{aligned}
$$

where the expression for the inverse quasipotential four-point propagator (86) and the quasipotential equation for the scattering matrix (88) were used.

We observe that the "." sign did not vanish on the last line of (103). This is due to the fact that although the inverse four-point quasipotential propagator was canceled, the constraint caused by it remained. To demonstrate this more effectively, we first undertake a very similar chain of steps to transform the left operator string of the effective current from (101) into $\{(1-U \Delta G) \ldots\}$, and then, keeping in mind that the quasipotential effective current is defined 
only on the submanifold of the total phase space, we write the final answer:

$$
J_{Q}^{\mu} \doteq(1-U \Delta G) J^{\mu}(1-\Delta G U)
$$

To see now that the current is conserved is straightforward. The substitution of the expression for the divergence of the Bethe-Salpeter current (51) into (104) gives

$$
\begin{aligned}
q_{\mu} J_{Q}^{\mu} \doteq & (1-U \Delta G)\left[e_{\Sigma}, \mathcal{G}^{-1}\right](1-\Delta G U) \\
\doteq & (1-U \Delta G) e_{\Sigma} \mathcal{G}^{-1}(1-\Delta G U) \\
& -(1-U \Delta G) \mathcal{G}^{-1} e_{\Sigma}(1-\Delta G U)
\end{aligned}
$$

Then the following transformations of the operator string right to the charge operator $e_{\Sigma}$ on the second line of (105) should be made:

$$
\begin{aligned}
\ldots \mathcal{G}^{-1}(1-\Delta G U) & =\ldots\left(G_{B S}^{-1}+V\right)(1-\Delta G U) \\
& =\ldots\left(G_{B S}^{-1}\left(1-\left(G_{B S}-g\right) U\right)+V(1-\Delta G U)\right) \\
& =\ldots\left(G_{B S}^{-1}-U+G_{B S}^{-1} g U+U\right) \\
& =\ldots G_{B S}^{-1}(1+g U) \\
& =\ldots G_{B S}^{-1} g \mathcal{G}_{Q}^{-1}
\end{aligned}
$$

where we used the expressions for the Bethe-Salpeter, (27), and the quasipotential, (86), inverse four-point propagators on the first and the fifth lines respectively, and the equation for the quasipotential kernel (66) on the third line. Making analogous transformation with the operator string left to the charge operator $e_{\Sigma}$ on the third line of (105), we write the contraction of the effective current with the external photon momentum as

$$
\begin{aligned}
q_{\mu} J_{Q}^{\mu} \doteq & -\mathcal{G}_{Q}^{-1} g G_{B S}^{-1} e_{\Sigma}(1-\Delta G U) \\
& +(1-U \Delta G) e_{\Sigma} G_{B S}^{-1} g \mathcal{G}_{Q}^{-1}
\end{aligned}
$$


which along with the quasipotential wave equation (92) guarantees that the current operator (95) is conserved,

$$
q_{\mu} \mathcal{J}^{\mu}=<\psi_{Q}\left|q_{\mu} J_{Q}^{\mu}\right| \psi_{Q}>=0 .
$$

One might consider actions undertaken between the fourth and fifth lines of the chain of equations (106) to be illegal since, instead of the general rule to possibly cancel all singularities, we inserted some singularities by representing $1+g U$ as $g \mathcal{G}_{Q}^{-1}$. Strictly speaking, this observation would be right. However, in this particular case, the singularity coming from $g$ was to be canceled by the zero associated with $G_{B S}^{-1}$. This means that we effectively did not touch the analytical structure of the expression in question and transformation (106) - made just to simplify the structure of the resulting expressionswas valid.

However, if we were more rigorous, we would write the divergence of the quasipotential effective current as

$$
\begin{aligned}
q_{\mu} J_{Q}^{\mu} \doteq & (1-U \Delta G) e_{\Sigma} G_{B S}^{-1}(1+g U) \\
& -(1+U g) G_{B S}^{-1} e_{\Sigma}(1-\Delta G U) .
\end{aligned}
$$

And then, considering the contraction of the current $\mathcal{J}^{\mu}$ with the photon momentum, we would write

$$
\begin{aligned}
q_{\mu} \mathcal{J}^{\mu}= & -<\psi_{Q} \mid\left\{(1+U g) G_{B S}^{-1} e_{\Sigma}(1-\Delta G U)\right. \\
& \left.-(1-U \Delta G) e_{\Sigma} G_{B S}^{-1}(1+g U)\right\} \mid \psi_{Q}> \\
= & -<\Gamma \mid g\left\{(1+U g) G_{B S}^{-1} e_{\Sigma}(1-\Delta G U)\right. \\
& \left.-(1-U \Delta G) e_{\Sigma} G_{B S}^{-1}(1+g U)\right\} g \mid \Gamma> \\
= & -<\Gamma \mid\left\{(g+g U g) G_{B S}^{-1} e_{\Sigma}(1-\Delta G U) g\right.
\end{aligned}
$$




$$
\begin{aligned}
& \left.-g(1-U \Delta G) e_{\Sigma} G_{B S}^{-1}(g+g U g)\right\} \mid \Gamma> \\
= & -<\Gamma \mid\left\{(1+g U) g G_{B S}^{-1} e_{\Sigma}(1-\Delta G U) g\right. \\
& \left.-g(1-U \Delta G) e_{\Sigma} G_{B S}^{-1} g(1+U g)\right\} \mid \Gamma>=0
\end{aligned}
$$

where we first used the quasipotential wave function definition (91) and the equations for the vertex functions in the form of (94) and (93) at the last step.

\subsubsection{Current Matrix Elements and Charge Conserva- tion}

The point to be noted here is that the quasipotential current is defined only on the reduced phase space (we used the $\doteq$ sign for it and for its contraction with $q_{\mu}$ in (107)) and it is not just a projection of the Bethe-Salpeter effective current $J^{\mu}$. Rather, it was carefully crafted in such a way that its matrix elements are the same as of $J^{\mu}$ (which is a natural reason why it is conserved). However, as it was stated above, this requirement had not been taken as a fundamental one and, hence, it is now to be checked. The proof, which could be considered as just a slight modification of transformations given in (110), is rather straightforward and is based only on the equations for the vertex functions (94) and (93) and the definitions of the quasipotential, (91), and the Bethe-Salpeter, (25), wave functions:

$$
\begin{aligned}
\mathcal{J}^{\mu} & =<\psi_{Q}\left|J_{Q}^{\mu}\right| \psi_{Q}> \\
& =<\Gamma\left|g(1-U \Delta G) J^{\mu}(1-\Delta G U) g\right| \Gamma> \\
& =<\Gamma\left|(-g U \Delta G+g) J^{\mu}(g-\Delta G U g)\right| \Gamma> \\
& =<\Gamma\left|(\Delta G+g) J^{\mu}(g+\Delta G)\right| \Gamma> \\
& =<\Gamma\left|G_{B S} J^{\mu} G_{B S}\right| \Gamma>
\end{aligned}
$$




$$
=<\psi\left|J^{\mu}\right| \psi>\text {. }
$$

We used the definition of the bound state wave functions to prove the equivalence of the current matrix elements in (111), but, due to Mandelstam [12] and as was already discussed in Section 2.3, the same equation should be held for the scattering wave functions. The explicit proof also exists, of course. Indeed, if we denote the on-shell plane waves by $\mid \mathbf{p}_{1}, \mathbf{p}_{2}>\left(<\mathbf{p}_{1}, \mathbf{p}_{2} \mid\right)$, then using the definition of the initial (final) state scattering wave functions with outgoing (incoming) spherical wave boundary conditions in the Bethe-Salpeter case

$$
\begin{aligned}
& \left|\psi>=\left(1-G_{B S} \mathcal{M}\right)\right| \mathbf{p}_{1}, \mathbf{p}_{2}> \\
& <\psi\left|=<\mathbf{p}_{1}, \mathbf{p}_{2}\right|\left(1-\mathcal{M} G_{B S}\right)
\end{aligned}
$$

and in the quasipotential case

$$
\begin{aligned}
& \left|\psi_{Q}>=(1-g \mathcal{M})\right| \mathbf{p}_{1}, \mathbf{p}_{2}> \\
& <\psi_{Q}\left|=<\mathbf{p}_{1}, \mathbf{p}_{2}\right|(1-\mathcal{M} g),
\end{aligned}
$$

we write the following series of transformations:

$$
\begin{aligned}
\mathcal{J}^{\mu}= & <\psi_{Q}\left|J_{Q}^{\mu}\right| \psi_{Q}> \\
= & <\mathbf{p}_{1}, \mathbf{p}_{2} \mid(1-\mathcal{M} g)(1-U \Delta G) J^{\mu} \\
& \times(1-\Delta G U)(1-g \mathcal{M}) \mid \mathbf{p}_{1}, \mathbf{p}_{2}> \\
= & <\mathbf{p}_{1}, \mathbf{p}_{2} \mid(1-\mathcal{M} g-U \Delta G+\mathcal{M} g U \Delta G) J^{\mu} \\
& \times(1-g \mathcal{M}-\Delta G U+\Delta G U g \mathcal{M}) \mid \mathbf{p}_{1}, \mathbf{p}_{2}> \\
= & <\mathbf{p}_{1}, \mathbf{p}_{2} \mid\left(1-\mathcal{M} G_{B S}+(\mathcal{M}-U+\mathcal{M} g U) \Delta G\right) J^{\mu} \\
& \times\left(1-G_{B S} \mathcal{M}+\Delta G(\mathcal{M}-U+U g \mathcal{M})\right) \mid \mathbf{p}_{1}, \mathbf{p}_{2}>
\end{aligned}
$$




$$
\begin{aligned}
& =<\mathbf{p}_{1}, \mathbf{p}_{2}\left|\left(1-\mathcal{M} G_{B S}\right) J^{\mu}\left(1-G_{B S} \mathcal{M}\right)\right| \mathbf{p}_{1}, \mathbf{p}_{2}> \\
& \left.=<\psi\left|J^{\mu}\right| \psi\right\rangle
\end{aligned}
$$

where we have also used the quasipotential equation for the scattering matrix.

This property of the current matrix elements preserves the equivalence of the normalization condition for the bound state vertex function and the charge conservation discussed in Section 2.6. The point here is that although we can not recover the zero momentum transfer limit of the quasipotential effective current $J_{Q}^{\mu}(0)$ from the Ward-Takahashi identities since-unlike the Bethe-Salpeter case - it generally has purely transverse components (cf. [16]), we still have an expression for the zero limit divergence of the effective current based on an analogous formula for the Bethe-Salpeter effective current (62):

$$
q_{\mu} J_{Q}^{\mu} \stackrel{q \rightarrow 0}{\longrightarrow}-(1-U \Delta G)\left(q_{\mu} e_{\Sigma} \frac{\partial \mathcal{G}^{-1}}{\partial P_{\mu}}\right)(1-\Delta G U) .
$$

While this expression does not uniquely determines the longitudinal part of the current because of the singularities associated with $\Delta G$, we still can proceed in the way similar to (111) to see that

$$
\begin{aligned}
q_{\mu} \mathcal{J}^{\mu} & =<\psi_{Q}\left|q_{\mu} J_{Q}^{\mu}\right| \psi_{Q}> \\
\stackrel{q \rightarrow 0}{\longrightarrow} & -<\psi_{Q}\left|(1-U \Delta G)\left(q_{\mu} e_{\Sigma} \frac{\partial \mathcal{G}^{-1}}{\partial P_{\mu}}\right)(1-\Delta G U)\right| \psi_{Q}> \\
& =-<\psi\left|q_{\mu} e_{\Sigma} \frac{\partial \mathcal{G}^{-1}}{\partial P_{\mu}}\right| \psi> \\
& =-2 q_{\mu} P^{\mu} e_{\Sigma}<\psi\left|\left(\mathcal{G}^{-1}\right)^{\prime}\right| \psi>=2 q_{\mu} P^{\mu} e_{\Sigma}
\end{aligned}
$$

because of the normalization condition (39). This implies the charge conservation condition (64).

Of course, omitting the discussion of the zero momentum transfer limit of the quasipotential effective current $J_{Q}^{\mu}(0)$, this effect could be seen directly, given that the current matrix elements do not change as it was shown in (111). 


\section{Chapter 4}

\section{Examples}

\subsection{Blankenbecler-Sugar Case}

The Blankenbecler-Sugar (BBS) approach [25, 37] (see also [38] for numerical results) was the first three-dimensional reduction technique in the history of quasipotential equations. This is not surprising since the BBS equation makes a logical link between the relativistic Bethe-Salpeter equation and the non-relativistic Lippman-Schwinger equation. To be more specific, the BBS equation can be constructed [1] as just a Lippman-Schwinger equation with the interaction modified in such a way that the resultant (BBS) equation gains the relativistic unitarity property. This modification prescription is referred to as "minimal relativity." After performing this procedure [1], one obtains that the quasipotential propagator written in the center of mass frame is given by equation (71) where function $f$ is equal to 1 (cf. Table 1 ),

$$
g_{B B S}=\frac{2 \pi}{E_{p}} \cdot \frac{\delta\left(p_{0}\right)}{4 E_{p}^{2}-W^{2}-i \epsilon},
$$


thus, the total phase space is reduced - the relative energy is not an independent variable and is restricted to zero.

We want to construct an explicit form of the BBS effective current [37]. An important observation [15] in this direction is that the constraint condition imposed upon the initial and final state relative momenta by the BBS propagator,

$$
p_{0}^{\prime}=p_{0}=0,
$$

is not compatible with the momentum conservation,

$$
p^{\prime}=p+q / 2
$$

where $q$ is the four-momentum of the photon. A natural way to deal with this is to assume that terms involving the momentum constraint both before and after interaction with the photon are equal to zero.

First, we consider terms coming from the impulse approximation part of the Bethe-Salpeter effective current. Since in the BBS approach the particles are treated symmetrically, we further simplify the procedure considering at the first step only those terms where the photon is attached to the first particle,

$$
\begin{aligned}
J_{\mathrm{BBS}, 1}^{\mu} \doteq & (1-U \Delta G) i J_{1}^{\mu} G_{2}^{-1}(1-\Delta G U) \\
\doteq & i J_{1}^{\mu} G_{2}^{-1} \\
& -U \Delta G i J_{1}^{\mu} G_{2}^{-1}-i J_{1}^{\mu} G_{2}^{-1} \Delta G U \\
& +U \Delta G i J_{1}^{\mu} G_{2}^{-1} \Delta G U
\end{aligned}
$$

Keeping in mind the meaning of the $\doteq$ sign (that the equation is considered only on the submanifold to which the total phase space was reduced), we immediately conclude that the term on the second line of the above expression 
is equal to zero. Terms on the third and fourth lines are to be expanded using (3) and (67),

$$
\begin{aligned}
J_{\mathrm{BBS}, 1}^{\mu} \doteq & U\left(i G_{1} G_{2}+g_{B B S}\right) i J_{1}^{\mu} G_{2}^{-1}+i J_{1}^{\mu} G_{2}^{-1}\left(i G_{1} G_{2}+g_{B B S}\right) U \\
& +U\left(i G_{1} G_{2}+g_{B B S}\right) i J_{1}^{\mu} G_{2}^{-1}\left(i G_{1} G_{2}+g_{B B S}\right) U \\
\doteq & -U G_{1} J_{1}^{\mu}-J_{1}^{\mu} G_{1} U \\
& +U g_{B B S} i J_{1}^{\mu} G_{2}^{-1}+i J_{1}^{\mu} G_{2}^{-1} g_{B B S} U+U g_{B B S} i J_{1}^{\mu} G_{2}^{-1} g_{B B S} U \\
& -U G_{1} J_{1}^{\mu} g_{B B S} U-U g_{B B S} J_{1}^{\mu} G_{1} U-U G_{1} i J_{1}^{\mu} G_{1} G_{2} U .
\end{aligned}
$$

All terms having the constrained relative momentum to both sides of the onebody current operator are vanishing on the fourth line, and the expression reduces to

$J_{\mathrm{BBS}, 1}^{\mu} \doteq-U G_{1} J_{1}^{\mu}-J_{1}^{\mu} G_{1} U-U G_{1} J_{1}^{\mu} g_{B B S} U-U g_{B B S} J_{1}^{\mu} G_{1} U-U G_{1} i J_{1}^{\mu} G_{1} G_{2} U$

The corresponding part of the current

$$
\mathcal{J}_{B B S, 1}^{\mu}=<\psi\left|J_{\mathrm{BBS}, 1}^{\mu}\right| \psi>
$$

is shown in Fig. 11, where the pairs of encircled crosses indicate that the relative energy is zero. Figs 12 and 13 illustrate the quasipotential wave equations (93) and (94) respectively. Graphically applying them to Fig. 11, we see that the diagrams on the first row of Fig. 11 cancel those on the second row, while the diagram on the third row becoming equivalent to the first diagram on Fig. 8. This is another demonstration of the already-known fact the current matrix elements do not change.

Recognizing that the sum of the matrix elements of the first four terms in (124) is equal to zero, Coester and Riska [37] chose to tackle the 
last term only. Introducing one more notation,

$$
\Delta_{1}=G_{1}+g_{B B S}
$$

the authors expressed the last term of (124) as

$$
G_{1} J_{1}^{\mu} G_{B S} \equiv-g_{B B S} J_{1}^{\mu} g_{B B S}-g_{B B S} J_{1}^{\mu} \Delta G+\Delta_{1} J_{1}^{\mu} G_{B S},
$$

and gave a reasonable interpretation of the resultant three terms. Obviously, the answer is the same since the last equation is a pure identity.

However, a slightly more important observation at this point would be that the equivalence of the matrix elements of the current in the form of (124) to the Bethe-Salpeter current matrix elements is of the purely theoretical importance since the use of the wave equations in the form of (93) or (94) is equivalent to moving from the reduced phase space to the total phase space, while the effective current was initially defined only on the zero relative energy subspace. The point here is that we do not need to leave this subspace to see that the current is conserved. Moreover, in any particular case, we can always check that the current is conserved directly. To demonstrate this, we consider the contraction of the external photon momentum with all terms in (124).

The first term gives

$$
-U G_{1} q_{\mu} J_{1}^{\mu} \doteq-U G_{1} e_{1} G_{1}^{-1}+U e_{1}
$$

It is convenient to consider the one-body propagators in the center of mass frame, where they have the following form:

$$
\begin{aligned}
G_{1}(p, W) & =\frac{1}{E_{p}^{2}-\left(\frac{W}{2}+p_{0}\right)^{2}-i \epsilon} \\
G_{2}(p, W) & =\frac{1}{E_{p}^{2}-\left(\frac{W}{2}-p_{0}\right)^{2}-i \epsilon} .
\end{aligned}
$$



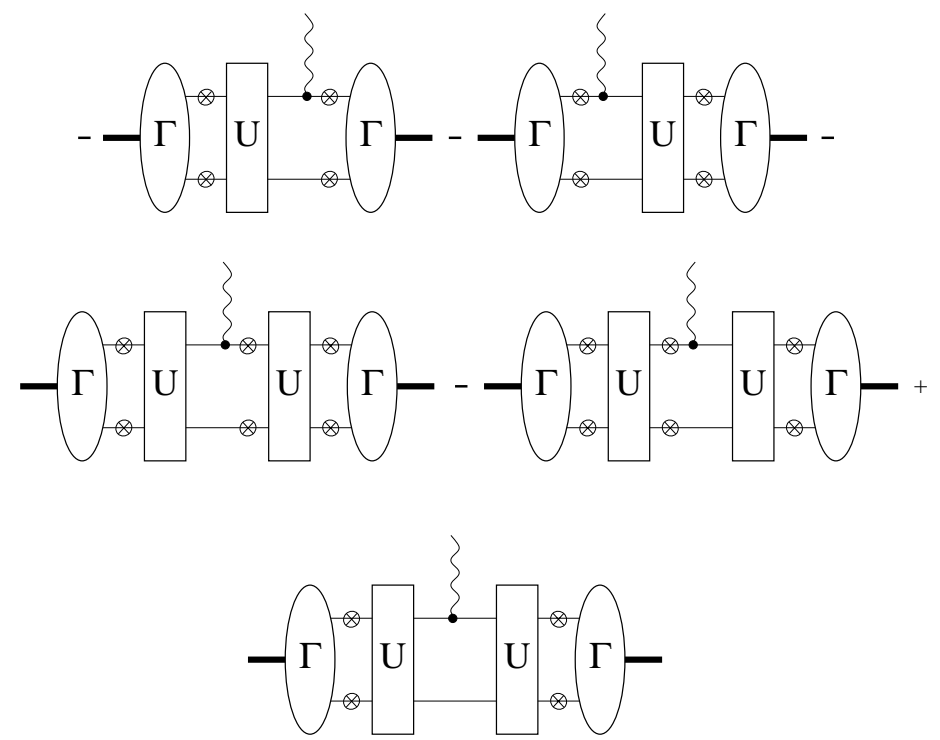

Figure 11: The IA part of the BBS current for the first particle.

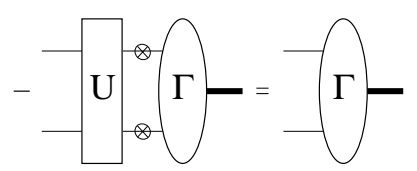

Figure 12: The quasipotential equation for $|\Gamma\rangle$.

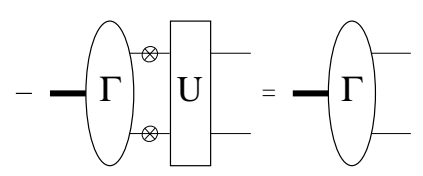

Figure 13: The quasipotential equation for $<\Gamma \mid$. 
The comparison of the above two equations with the BBS propagator in the center of mass frame (119) yields

$$
G_{1} \doteq 2 g_{B B S} \doteq G_{2}
$$

if we take into account that the momentum space states normalization condition (10) should be rewritten for the reduced phase space as

$$
1=\int \frac{d^{3} p}{(2 \pi)^{3} 2 E_{p}}|\mathbf{p}><\mathbf{p}|
$$

Thus, equation (128) becomes

$$
-U G_{1} q_{\mu} J_{1}^{\mu} \doteq-\frac{1}{2} U G_{1} e_{1} g_{B B S}^{-1}+U e_{1} .
$$

In a similar fashion, for the second term of (124):

$$
-q_{\mu} J_{1}^{\mu} G_{1} U \doteq-e_{1} U+\frac{1}{2} g_{B B S}^{-1} e_{1} G_{1} U
$$

Using that

$$
G_{1}^{-1} g_{B B S} \doteq \frac{\dot{ }}{2} \doteq G_{2}^{-1} g_{B B S},
$$

where the dot in the numerator is left to remind that the relative momentum is constrained, we write the contraction of the third and fourth terms of (124) as

$$
\begin{aligned}
-U G_{1} q_{\mu} J_{1}^{\mu} g_{B B S} U & \doteq-\frac{1}{2} U G_{1} e_{1} \cdot U+U e_{1} g_{B B S} U \\
-U g_{B B S} q_{\mu} J_{1}^{\mu} G_{1} U & \doteq-U g_{B B S} e_{1} U+\frac{1}{2} U \cdot e_{1} G_{1} U
\end{aligned}
$$

And, finally, for the last term:

$$
-U G_{1} i q_{\mu} J_{1}^{\mu} G_{1} G_{2} U \doteq-U G_{1} i e_{1} G_{2} U+U i e_{1} G_{1} G_{2} U
$$

The BBS approach treats both particles completely symmetrically. Therefore, the $J_{\mathrm{BBS}, 2}^{\mu}$ part of the current could be drawn to result in a picture 
similar to Fig. 11 but with the photon attached to the nucleon represented by the bottom line (if we agree that the nucleon represented by the bottom line is the second particle). The algebraic expression for the current $J_{\mathrm{BBS}, 2}^{\mu}$ has the form given in (124) with indices 1 and 2 transposed. So do equations (133134, 136-138) arising during the calculation of $q_{\mu} J_{\mathrm{BBS}, 2}^{\mu}$. Summing all these equations - along with those with transposed indices, - we obtain the following expression for the divergence of the impulse approximation part of the BBS effective current:

$$
\begin{aligned}
q_{\mu} J_{\mathrm{BBS}, \mathrm{IA}}^{\mu} \doteq & q_{\mu}\left(J_{\mathrm{BBS}, 1}^{\mu}+J_{\mathrm{BBS}, 2}^{\mu}\right) \\
\doteq & -\frac{1}{2} U\left(G_{1} e_{1}+G_{2} e_{2}\right) g_{B B S}^{-1}+g_{B B S}^{-1}\left(e_{1} G_{1}+e_{2} G_{2}\right) U \frac{1}{2} \\
& -\left[e_{\Sigma}, U\right] \\
& -\frac{1}{2} U\left(G_{1} e_{1}+G_{2} e_{2}\right) \cdot U+U \cdot\left(e_{1} G_{1}+e_{2} G_{2}\right) U \frac{1}{2} \\
& +U\left[e_{\Sigma}, g_{B B S}\right] U+U i\left[e_{1}, G_{1}\right] G_{2} U+U i\left[e_{2}, G_{2}\right] G_{1} U \\
\doteq & -\frac{1}{2} U\left(G_{1} e_{1}+G_{2} e_{2}\right)\left(g_{B B S}^{-1}+\cdot U\right) \\
& +\left(g_{B B S}^{-1}+U \cdot\right)\left(e_{1} G_{1}+e_{2} G_{2}\right) U \frac{1}{2} \\
& -\left[e_{\Sigma}, U\right]-U\left[e_{\Sigma}, \Delta G\right] U \\
\doteq & \mathcal{G}_{B B S}^{-1}\left(e_{1} G_{1}+e_{2} G_{2}\right) U \frac{1}{2}-\frac{1}{2} U\left(G_{1} e_{1}+G_{2} e_{2}\right) \mathcal{G}_{B B S}^{-1} \\
& -\left[e_{\Sigma}, U\right]-U\left[e_{\Sigma}, \Delta G\right] U .
\end{aligned}
$$

The last two terms are canceled by the divergence of the exchange part of the BBS effective current,

$$
J_{\mathrm{BBS}, \mathrm{ex}}^{\mu} \doteq(1-U \Delta G) J_{\mathrm{ex}}^{\mu}(1-\Delta G U)
$$

Indeed,

$$
q_{\mu} J_{\mathrm{BBS}, \mathrm{ex}}^{\mu} \doteq(1-U \Delta G) q_{\mu} J_{\mathrm{ex}}^{\mu}(1-\Delta G U)
$$




$$
\begin{aligned}
& \doteq(1-U \Delta G)\left[e_{\Sigma}, V\right](1-\Delta G U) \\
& \doteq(1-U \Delta G) e_{\Sigma}(V-V \Delta G U)-(V-U \Delta G V) e_{\Sigma}(1-\Delta G U) \\
& \doteq(1-U \Delta G) e_{\Sigma} U-U e_{\Sigma}(1-\Delta G U) \\
& \doteq\left[e_{\Sigma}, U\right]+U\left[e_{\Sigma}, \Delta G\right] U
\end{aligned}
$$

where we have used the equation for the quasipotential kernel (66).

The last equation reproduces the basic result of Coester and Riska [37]. Their "minimal" exchange current was derived to have the following form:

$$
J_{\mathrm{BBS}-\mathrm{CR}, \mathrm{ex}}^{\mu} \doteq \frac{q^{\mu}}{q^{2}}\left(\left[e_{\Sigma}, U\right]+U\left[e_{\Sigma}, \Delta G\right] U\right)
$$

The word "minimal" stands to indicate that only the longitudinal part of the current was obtained. Equation (141) demonstrates that the longitudinal part of the current $J_{\mathrm{BBS}-\mathrm{CR} \text {,ex }}^{\mu}$ in (142) is equivalent to that one of $J_{\mathrm{BBS} \text {,ex }}^{\mu}$ in (140). Combining the results of (139) and (141), we write the divergence of the BBS effective current as

$$
q_{\mu} J_{\mathrm{BBS}}^{\mu} \doteq \mathcal{G}_{B B S}^{-1}\left(e_{1} G_{1}+e_{2} G_{2}\right) U \frac{1}{2}-\frac{1}{2} U\left(G_{1} e_{1}+G_{2} e_{2}\right) \mathcal{G}_{B B S}^{-1}
$$

which along with the wave equation (92) implies that the current is conserved.

The essence of the question is that this result immediately follows from our generic expression (107):

$$
\begin{aligned}
q_{\mu} J_{\mathrm{BBS}}^{\mu} \doteq & -\mathcal{G}_{B B S}^{-1} g_{B B S} G_{B S}^{-1} e_{\Sigma}(1-\Delta G U) \\
& +(1-U \Delta G) e_{\Sigma} G_{B S}^{-1} g_{B B S} \mathcal{G}_{B B S}^{-1} \\
\doteq & -\mathcal{G}_{B B S}^{-1} g_{B B S} G_{B S}^{-1} e_{\Sigma}-\mathcal{G}_{B B S}^{-1} g_{B B S} G_{B S}^{-1} e_{\Sigma} g_{B B S} U \\
& +\mathcal{G}_{B B S}^{-1} g_{B B S} G_{B S}^{-1} e_{\Sigma} G_{B S} U-U G_{B S} e_{\Sigma} G_{B S}^{-1} g_{B B S} \mathcal{G}_{B B S}^{-1} \\
& +U g_{B B S} e_{\Sigma} G_{B S}^{-1} g_{B B S} \mathcal{G}_{B B S}^{-1}+e_{\Sigma} G_{B S}^{-1} g_{B B S} \mathcal{G}_{B B S}^{-1} .
\end{aligned}
$$


All terms on the third and fifth lines vanish since the momentum can not be constrained on both sides of the charge operators (momentum conservation). The algebraic transformations for the terms on the fourth line are:

$$
\begin{aligned}
q_{\mu} J_{\mathrm{BBS}}^{\mu} \doteq & \mathcal{G}_{B B S}^{-1} g_{B B S} G_{B S}^{-1} e_{\Sigma} G_{B S} U-U G_{B S} e_{\Sigma} G_{B S}^{-1} g_{B B S} \mathcal{G}_{B B S}^{-1} \\
\doteq & \mathcal{G}_{B B S}^{-1} g_{B B S} G_{1}^{-1} G_{2}^{-1}\left(e_{1}+e_{2}\right) G_{1} G_{2} U \\
& -U G_{1} G_{2}\left(e_{1}+e_{2}\right) G_{1}^{-1} G_{2}^{-1} g_{B B S} \mathcal{G}_{B B S}^{-1} \\
\doteq & \mathcal{G}_{B B S}^{-1} g_{B B S}\left(G_{1}^{-1} e_{1} G_{1}+G_{2}^{-1} e_{2} G_{2}\right) U \\
& -U\left(G_{1} e_{1} G_{1}^{-1}+G_{2} e_{2} G_{2}^{-1}\right) g_{B B S} \mathcal{G}_{B B S}^{-1}
\end{aligned}
$$

which produces the already derived answer (143) after using equation (135) where appropriate.

\subsection{Gross Case}

The Gross equation [30, 21] (numerical results can be found in [23]) is suited especially well for description of the two-body system with one heavy particle. As was shown in [4], there is a delicate cancellation of the boson pole contributions coming from the box and crossed box diagrams in the Bethe-Salpeter approach. This cancellation is exact as soon as the mass of the second particle goes to infinity. Another effect is that in this limit the sum of the box and crossed box diagrams is equivalent to placing the heavy particle on-shell. Since the Gross propagator is constructed in such a way that it is equivalent to the Bethe-Salpeter propagator with one of the particles on-shell, the OBE approximation in this approach effectively counts not only contributions of ladder diagrams but contributions coming from crossed ladder diagrams as well. That is why, unlike the Bethe-Salpeter OBE approximation, the OBE 
approximation in the Gross case produces the correct one-body limit (another quasipotential that was constructed to explicitly respect the one-body limit is by Wallace and Mandelzweig [33, 34]).

More care should be taken considering two-body systems comprising nonzero spin particles. The spin structure may interfere with and break the fine cancellation effects described above.

Also, an obvious disadvantage of this method becomes crucial when considering two-body systems consisting of identical particles. Since the Gross equation does not treat particles symmetrically, an explicit symmetrization technique [23] should be applied to the system in this case.

As in the previous section, our primary task is to construct the effective current for the Gross equation from the generic results obtained before and to see how the matrix elements of this current are related to those in the fundamental paper [21] devoted to the method.

As follows from equation (72) and Table 1, the Gross propagator in the center of mass frame is equal to

$$
g_{G}(p ; W)=\frac{2 \pi}{2 E_{p}} \delta\left(p_{0}-\left(E_{p}-W / 2\right)\right) \frac{1}{W\left(2 E_{p}-W-i \epsilon\right)} .
$$

To see that this is equivalent to placing the first particle on-shell is easy. The first factor in (146), $\frac{2 \pi}{2 E_{p}}$, is absorbed into the normalization condition (132); the $\delta$-function restricts the relative momenta such that the first particle is onshell; and the last factor $\frac{1}{W\left(2 E_{p}-W-i \epsilon\right)}$ is just equal to the one-body propagator for the second particle,

$$
G_{2}(p ; W)={ }_{c . m} \cdot \frac{1}{E_{p}^{2}-\left(W / 2-p_{0}\right)^{2}-i \epsilon},
$$

where $p_{0}$ is given by the constraint,

$$
p_{0}=E_{p}-W / 2 \text {. }
$$


In our notation, all the above is simply written as

$$
g_{G} \doteq G_{2}
$$

Due to asymmetry embedded in the Gross approach, we have to tackle pieces of the impulse approximation part of the effective current for the two particles separately. We start with the first particle:

$$
\begin{aligned}
J_{\mathrm{G}, 1}^{\mu} \doteq & (1-U \Delta G) i J_{1}^{\mu} G_{2}^{-1}(1-\Delta G U) \\
\doteq & \left(1+U\left(i G_{1} G_{2}+\cdot G_{2}\right)\right) i J_{1}^{\mu} G_{2}^{-1}\left(1+\left(i G_{1} G_{2}+G_{2} \cdot\right) U\right) \\
\doteq & i J_{1}^{\mu} G_{2}^{-1}+U \cdot G_{2} i J_{1}^{\mu} G_{2}^{-1}+i J_{1}^{\mu} G_{2}^{-1} G_{2} \cdot U+U \cdot G_{2} i J_{1}^{\mu} G_{2}^{-1} G_{2} \cdot U \\
& +U i G_{1} G_{2} i J_{1}^{\mu} G_{2}^{-1}+i J_{1}^{\mu} G_{2}^{-1} i G_{1} G_{2} U \\
& +U i G_{1} G_{2} i J_{1}^{\mu} G_{2}^{-1} G_{2} \cdot U+U \cdot G_{2} i J_{1}^{\mu} G_{2}^{-1} i G_{1} G_{2} U \\
& +U i G_{1} G_{2} i J_{1}^{\mu} G_{2}^{-1} i G_{1} G_{2} U
\end{aligned}
$$

where "." indicates that the relative momentum is constrained. Again, the momentum conservation is not compatible with a particle being on-shell both before and after interaction with a photon. Therefore, all terms on the third line are vanishing from the above expression, and what remains is very similar to (124):

$$
J_{\mathrm{G}, 1}^{\mu} \doteq-U G_{1} J_{1}^{\mu}-J_{1}^{\mu} G_{1} U-U G_{1} J_{1}^{\mu} g_{G} U-U g_{G} J_{1}^{\mu} G_{1} U-U G_{1} i J_{1}^{\mu} G_{1} G_{2} U
$$

The Feynman diagrams for the corresponding matrix elements are given by Fig. 14, where crosses indicate that the particle is on-shell. One can easily see again that the matrix elements of the current do not change.

An interesting observation, however, is that the divergence of the effective current for the first particle vanishes in the strong sense (on the operator level). 


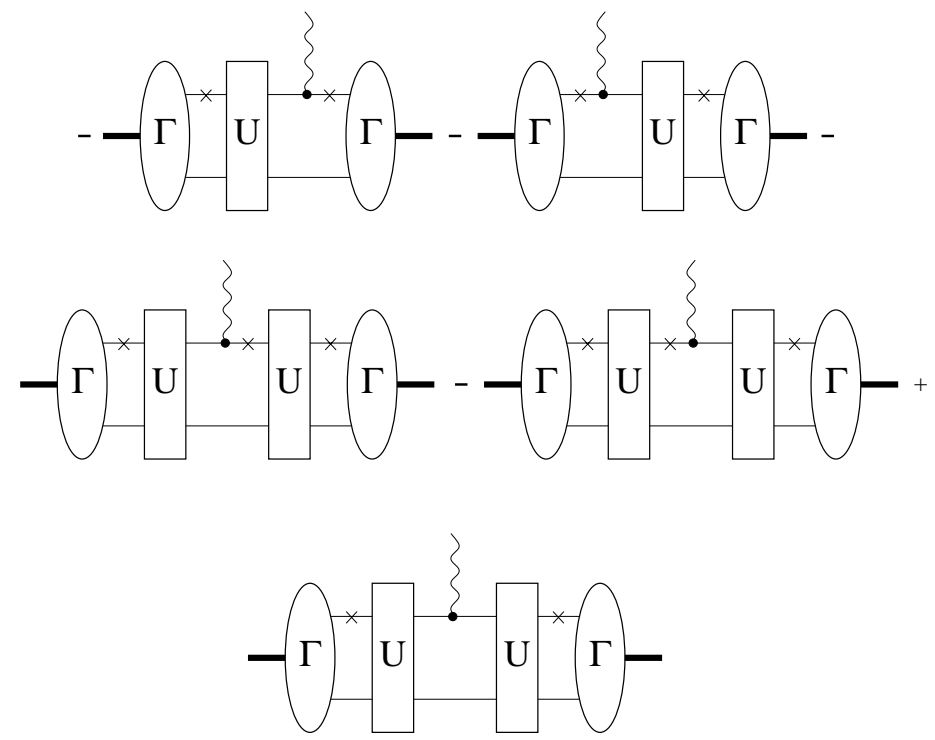

Figure 14: The IA part of the Gross current for the first particle.

This is not surprising since the particle is on-shell:

$$
G_{1}^{-1} \doteq 0
$$

Considering the contraction of the photon momentum with the first term in (151), we see that:

$$
-U G_{1} q_{\mu} J_{1}^{\mu} \doteq-U G_{1} e_{1} G_{1}^{-1}+U G_{1} G_{1}^{-1} e_{1} \doteq U e_{1}
$$

because of (152). Similarly, for the second term:

$$
-q_{\mu} J_{1}^{\mu} G_{1} U \doteq-e_{1} U
$$

Using of (152) again for the third and forth terms of (151) yields

$$
-q_{\mu}\left(U G_{1} J_{1}^{\mu} g_{G} U+U g_{G} J_{1}^{\mu} G_{1} U\right) \doteq U\left[e_{1}, g_{G}\right] U
$$


The last term gives

$$
-U G_{1} i q_{\mu} J_{1}^{\mu} G_{1} G_{2} U \doteq-U\left[e_{1}, G_{B S}\right] U
$$

and the divergence of the current $J_{\mathrm{G}, 1}^{\mu}$ becomes

$$
q_{\mu} J_{\mathrm{G}, 1}^{\mu} \doteq-\left[e_{1}, U\right]-U\left[e_{1}, \Delta G\right] U
$$

The divergence of the exchange part of the effective current (and the current itself) is given by the equation that looks identically to (141) (but $\Delta G$ and $U$ are different, of course). The part of this divergence corresponding to the first particle,

$$
q_{\mu} J_{\mathrm{G}, \mathrm{ex}, 1}^{\mu} \doteq\left[e_{1}, U\right]+U\left[e_{1}, \Delta G\right] U
$$

cancels (157).

Some special care should be taken considering the effective current for the second particle (in the first particle on-shell cases). This was done in [16]. The answer (cf. Fig. 15) is

$$
J_{\mathrm{G}, 2}^{\mu} \doteq J_{2}^{\mu}-U G_{2} J_{2}^{\mu} g_{G} U-U G_{2} i J_{2}^{\mu} G_{2} G_{1} U
$$

The current is obviously conserved. Indeed, it can be rewritten as

$$
J_{\mathrm{G}, 2}^{\mu} \doteq J_{2}^{\mu}+U G_{2} J_{2}^{\mu} \Delta G U
$$

and, hence, the divergence of the current yields

$$
q_{\mu} J_{\mathrm{G}, 2}^{\mu} \doteq\left[e_{2}, G_{2}^{-1}\right]-U\left[e_{2}, \Delta G\right] U
$$

Given that the divergence of $J_{\mathrm{G}, 1}^{\mu}$ vanish in the strong sense and using the expression for the divergence of the exchange current corresponding to the second particle (cf. 158)

$$
q_{\mu} J_{\mathrm{G}, \mathrm{ex}, 2}^{\mu} \doteq\left[e_{2}, U\right]+U\left[e_{2}, \Delta G\right] U
$$




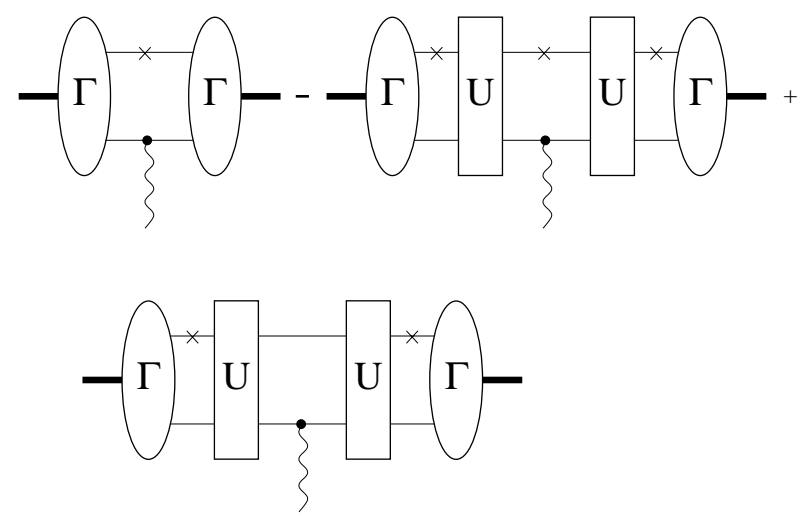

Figure 15: The IA part of the Gross current for the second particle.

we conclude that the divergence of $J_{\mathrm{G}}^{\mu}$ is linear with respect to $U$ :

$$
q_{\mu} J_{\mathrm{G}}^{\mu} \doteq\left[e_{2}, G_{2}^{-1}+U\right] \doteq\left[e_{2}, \mathcal{G}_{G}^{-1}\right]
$$

One of the most important consequences of this conclusion is that truncation does not introduce any additional difficulties and can be performed [16] very much like in the Bethe-Salpeter case where the divergence of the current is also linear with respect to the kernel (cf. the next chapter).

A very important observation is that this current (as well as the BBS current) could be obtained directly from the Bethe-Salpeter current using the wave equation (or, to be more general, using the Bethe-Salpeter equation, which is reduced to the wave equation on the scattering and bound states). The current is a subject to the following three obvious requirements: it should be conserved, its matrix elements should be equal to the matrix elements of the Bethe-Salpeter current and the both initial and final momenta should be properly constrained (a quasipotential effective current can be defined only on the submanifold, to which the total phase space is reduced). 


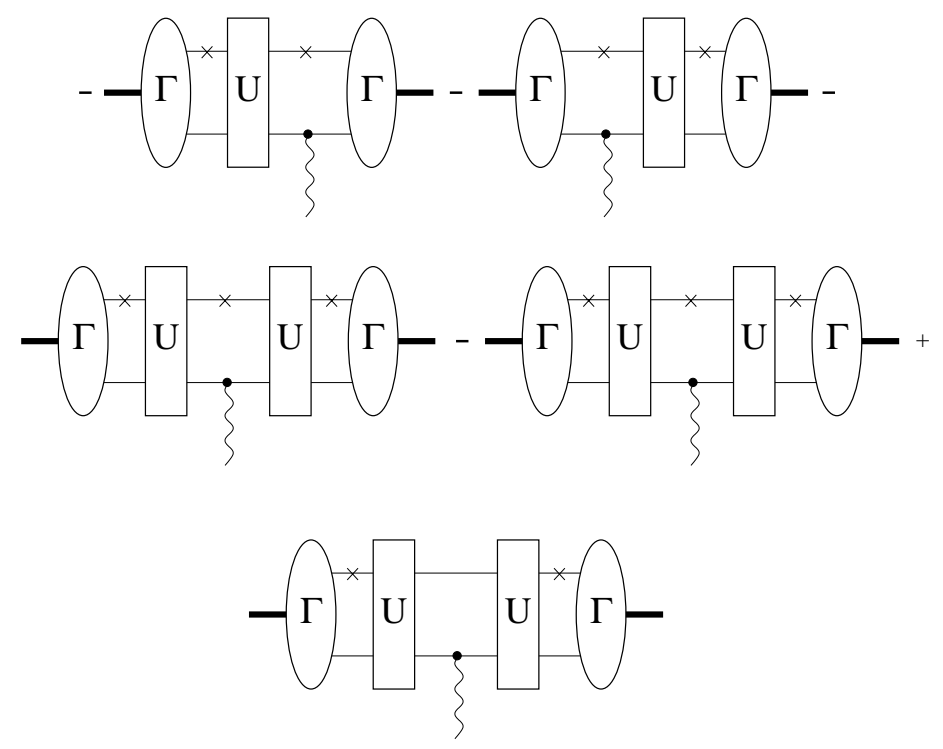

Figure 16: Current $J_{\mathrm{GM}, 2}^{\mu}$.

Consider another current depicted on Fig. 16. It can be expressed as

$$
J_{\mathrm{GM}, 2}^{\mu} \doteq-U G_{2} J_{2}^{\mu}-J_{2}^{\mu} G_{2} U-U G_{2} J_{2}^{\mu} g_{G} U-U g_{G} J_{2}^{\mu} G_{2} U-U G_{1} G_{2} i J_{2}^{\mu} G_{2} U
$$

and it does satisfy all three requirements above. The third one is fulfilled due to the presence of the "ㄹ" sign. The second requirements is also obviously satisfied since Fig. 16 (as well as Fig. 15, of course) is the most transparent indication that the matrix elements do not change. And the current conservation can be easily checked, too. Indeed, contraction of the external photon momentum with the first two terms of (164) gives

$$
\begin{aligned}
-U G_{2} q_{\mu} J_{2}^{\mu}-q_{\mu} J_{2}^{\mu} G_{2} U & \doteq-U G_{2} e_{2} G_{2}^{-1}+U e_{2}-e_{2} U+G_{2}^{-1} e_{2} G_{2} U \\
& \doteq g_{G}^{-1} e_{2} G_{2} U-U G_{2} e_{2} g_{G}^{-1}-\left[e_{2}, U\right]
\end{aligned}
$$


The same procedure for the third term yields

$$
-2 U \cdot G_{2} q_{\mu} J_{2}^{\mu} G_{2} \cdot U \doteq 2 U \cdot\left[e_{2}, G_{2}\right] \cdot U \doteq 2 U\left[e_{2}, g_{G}\right] U
$$

And, for the last term:

$$
-U G_{2} i q_{\mu} J_{2}^{\mu} G_{2} G_{1} U \doteq U i G_{1}\left[e_{2}, G_{2}\right] U \doteq-U\left[e_{2}, G_{B S}\right] U
$$

Summing this all up, we write the divergence of the current $J_{\mathrm{GM}, 2}^{\mu}$ as

$$
\begin{aligned}
q_{\mu} J_{\mathrm{GM}, 2}^{\mu} \doteq & g_{G}^{-1} e_{2} G_{2} U-U G_{2} e_{2} g_{G}^{-1}-\left[e_{2}, U\right] \\
& +2 U\left[e_{2}, g_{G}\right] U-U\left[e_{2}, G_{B S}\right] U \\
\doteq & g_{G}^{-1} e_{2} G_{2} U-U G_{2} e_{2} g_{G}^{-1}-\left[e_{2}, U\right] \\
& +U \cdot e_{2} G_{2} U-U G_{2} e_{2} \cdot U-U\left[e_{2}, \Delta G\right] U \\
\doteq & \mathcal{G}_{G}^{-1} e_{2} G_{2} U-U G_{2} e_{2} \mathcal{G}_{G}^{-1} \\
& -\left[e_{2}, U\right]-U\left[e_{2}, \Delta G\right] U .
\end{aligned}
$$

The last two terms are canceled by the corresponding part of the divergence of the exchange current, and the wave equation for the terms on the fifth line guarantees the current conservation.

However, current (164) is not "minimal" in the following sense. We notice that $J_{\mathrm{GM}, 2}^{\mu}$ is equivalent to the impulse approximation part of the effective current for the first particle (151) with indices 1 and 2 transposed. And now comparing (151) with the corresponding expression for the BBS case (124) and also remembering that, in the BBS case, the impulse approximation current for the second particle is given by the same expression (124) (with $\{1 \leftrightarrow 2\}$ ), we conclude that

$$
J_{\mathrm{eff}, \mathrm{a}}^{\mu} \doteq-U G_{a} J_{a}^{\mu}-J_{a}^{\mu} G_{a} U-U G_{a} J_{a}^{\mu} g U-U g J_{a}^{\mu} G_{a} U-U G_{a} i J_{a}^{\mu} G_{a} G_{b} U
$$




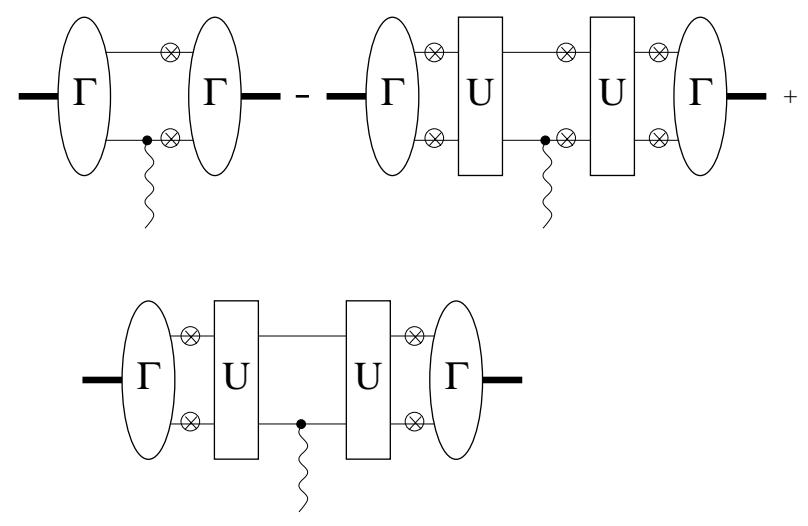

Figure 17: In any off-shell case, the current can not be reduced.

where pair of indices $(a, b)$ is a transposition of the pair $(1,2)$, is a generic form for the impulse approximation part of the effective current for the ath particle (compare Figs. 11, 14 and 16) unless the bth particle is on-shell. In this case, the current can be further reduced to the form of (159) (Fig. 15), which coincides with what the Mandelstam formalism gives due to [16].

An attempt to represent the current similarly to Fig. 15 in off-shell cases would lead to what is shown on Fig. 17. This current is not acceptable since it does not satisfy the third requirement - the final momentum of the first term is not properly constrained. This is obviously related to the fact that the constraint condition does not touch the momentum of the second particle in on-shell cases only.

It is very instructive to discuss the connection of these results to the original results obtained by Gross and Riska in [21]. Their total effective current was given by

$$
J_{\mathrm{GR}}^{\mu} \doteq-J_{1}^{\mu} G_{1} V-V G_{1} J_{1}^{\mu}+J_{2}^{\mu}+J_{\mathrm{ex}}^{\mu}
$$

where $J_{\mathrm{ex}}^{\mu}$ is the same exchange current as for the Bethe-Salpeter case (its 


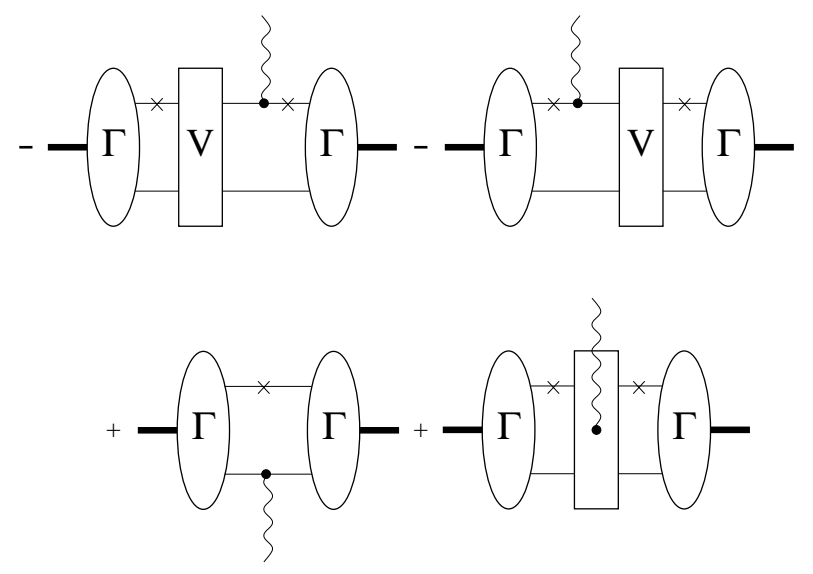

Figure 18: The original Gross effective current.

divergence is as in (49)). If we look at Fig. 18 representing the current (170) matrix elements, we immediately conclude that these matrix elements differ from those of the Bethe-Salpeter current on Fig. 8. This gives rise for a number of obvious problems. Normalization conditions for the vertex functions restricted to the reduced phase space [22] is now to be checked again. The form of the wave equation used,

$$
\left|\Gamma>\doteq-V \cdot G_{2}\right| \Gamma>,
$$

coincides with the correct form of the wave equation (93) in the case of OBE approximation only, when equation (66) reduces to

$$
U^{(1)}=V^{(1)},
$$

where upper indices indicate the number of boson exchanges.

This also sheds some light on appearances of $V$ (instead of $U$ ) in (170). The current given by (170) is conserved only when the OBE approximation 
is in effect. The proof of the current conservation given in [21] looks in our notation as

$$
\begin{aligned}
q_{\mu} J_{\mathrm{GR}}^{\mu} & \doteq-e_{1} V+G_{1}^{-1} e_{1} G_{1} V-V G_{1} e_{1} G_{1}^{-1}+V e_{1}+\left[e_{2}, G_{2}^{-1}\right]+\left[e_{\Sigma}, V\right] \\
& \doteq-\left[e_{1}, V\right]+\left[e_{2}, G_{2}^{-1}\right]+\left[e_{1}+e_{2}, V\right] \\
& \doteq\left[e_{2}, G_{2}^{-1}+V\right]
\end{aligned}
$$

where (152) was used between the first and the second lines. Since the wave equation (171) is valid only for the OBE approximation, only in this case do the matrix elements of the divergence of the current in (173) vanish.

However, the original Gross effective current on Fig. 18 possesses a great advantage. Its construction can be (and was) governed by the clear intuitive motivations. We take all diagrams on Fig. 8 and place the first particle onshell where possible. Since we can place the first particle on-shell both before and after its interaction with a photon, the first diagram on Fig. 8 gives rise to two, which after applying to them the wave equation in the form of (171) become the first and the second diagrams on Fig. 18. 


\section{Chapter 5}

\section{Truncation Issues}

One of the powerful features of our expression for the generic effective current (104) is that it allows one to easily resolve difficulties associated with truncation procedures in the generic quasipotential approach. All the results above can not be considered complete unless they are accompanied by the clear view of what happens when the truncation is turned on.

As was already mentioned, all quasipotential approaches are equivalent to the Bethe-Salpeter approach only when the values of both the quasipotential and the Bethe-Salpeter kernels are exact. This equivalence is, therefore, of purely theoretical importance since as soon as the kernels are truncated, the results differ. And as we already noticed in Section 3.1.1, results differ in such a way that all quasipotential approaches in the ladder approximation provide better convergence properties than the Bethe-Salpeter ladder approximation. 


\subsection{Bethe-Salpeter Case}

Truncation does not introduce any complications in the Bethe-Salpeter case. The kernel can be truncated at any number of boson exchanges. We can represent the kernel $V$ as

$$
V=\sum_{n>0} V^{(n)}
$$

where $V^{(n)}$ stands for the $n$-boson exchange contribution. If we look at Fig. 2, we identify $V^{(1)}$ with the first diagram, $V^{(2)}$ — with the second one, and $V^{(3)}$ with the third, fourth and fifth. The notation we use for the up-to- $N$-boson exchange contribution is

$$
V^{[N]}=\sum_{n>0}^{N} V^{(n)} .
$$

That is, $V^{[3]}$ is represented by all diagrams on Fig. 2. The Bethe-Salpeter current (41) can be expanded then in a consistent manner

$$
J^{\mu}=\sum_{n \geq 0} J^{\mu(n)}
$$

where $J^{\mu(0)}$ is naturally put to

$$
J^{\mu(0)}=J_{\mathrm{IA}}^{\mu}
$$

and the exchange current is expanded as

$$
J_{\mathrm{ex}}^{\mu}=\sum_{n>0} J_{\mathrm{ex}}^{\mu(n)}
$$

Here, term $J_{\mathrm{ex}}^{\mu(1)}$ corresponds to the photon interacting with the (charged) boson on the first diagram of Fig. 2 whereas $J_{\mathrm{ex}}^{\mu(2)}$ is actually four (two-in the case of uncharged bosons) terms coming from the photon interaction with two bosons and two internal fermions on the second diagram of Fig. 2, and $J_{\text {ex }}^{\mu(3)}$ 
is represented by twenty one term - seven terms per each of the last three diagrams on Fig. 2.

The current conservation is not perturbed in the presence of truncation since

$$
\begin{aligned}
q_{\mu} J^{\mu(0)} & =\left[e_{\Sigma}, G_{B S}^{-1}\right], \\
q_{\mu} J_{\mathrm{ex}}^{\mu(n)} & =\left[e_{\Sigma}, V^{(n)}\right], \quad n>0,
\end{aligned}
$$

as it can be seen from the explicit proof of the Ward-Takahashi identity for the exchange current (49) given in [21]. The form of the wave equation (28) in the presence of truncation is

$$
V^{[N]} \mid \psi^{[N]}>=0
$$

where, anticipating future benefits of the notation, we have just started using

$$
V^{[N]}=\sum_{n \geq 0}^{N} V^{(n)}
$$

instead of (175), and where we put

$$
V^{(0)}=G_{B S}^{-1}
$$

The wave equation (181) implies then that the current $J^{\mu[N]}$ is conserved:

$$
\begin{aligned}
q_{\mu} \mathcal{J}^{\mu[N]} & =<\psi^{[N]}\left|q_{\mu} J^{\mu[N]}\right| \psi^{[N]}>=<\psi^{[N]}\left|q_{\mu} \sum_{n \geq 0}^{N} J^{\mu(n)}\right| \psi^{[N]}> \\
& =<\psi^{[N]} \mid q_{\mu}\left(J_{\mathrm{IA}}^{\mu}+\sum_{n>0}^{N} J_{\mathrm{ex}}^{\mu(n)} \mid \psi^{[N]}>\right. \\
& =<\psi^{[N]}\left|\left[e_{\Sigma}, G_{B S}^{-1}+\sum_{n>0}^{N} V^{(n)}\right]\right| \psi^{[N]}> \\
& =<\psi^{[N]}\left|\left[e_{\Sigma}, V^{[N]}\right]\right| \psi^{[N]}>=0 .
\end{aligned}
$$




\subsection{Generic Quasipotential}

It is clear that the situation is not so simple in the quasipotential case. The quasipotential effective current $J_{Q}^{\mu}(104)$ contains linear, quadratic and cubic (with respect to expansion) terms since both the Bethe-Salpeter current $J^{\mu}$ and the quasipotential kernel $U$ are to be expanded. Therefore, unlike the Bethe-Salpeter case, the divergence of the $n$-boson exchange piece of the current, $J_{Q}^{\mu(n)}$, is not given by an expression similar to the expression for the divergence of the non-truncated current. This breaks the conservation of the truncated current. Some procedure is required to extract contributions giving the conserved part of the current truncated at some particular number of boson exchanges and to identify "extraneous" parts of the current we have to add or subtract to restore the current conservation. This is the ultimate purpose of this section.

\subsubsection{Kernel Expansion}

We start with the discussion of the $n$-boson contributions to the quasipotential effective current, $J_{Q}^{\mu(n)}$. The expansion of $J^{\mu}$ in (104) is obviously given by and has the same meaning as in (176). Like the Bethe-Salpeter kernel $V$, the quasipotential kernel $U$ can be also represented as a series

$$
U=\sum_{n>0} U^{(n)}
$$

Furthermore, each term in this series can be expressed as a function of $V^{(i)}$ 's $(i \leq n)$ using the quasipotential equation (66). Indeed, consider the expanded form of equation (66)

$$
\sum_{n>0} U^{(n)}=\sum_{n>0} V^{(n)}-\sum_{i>0} V^{(i)} \Delta G \sum_{j>0} U^{(j)}
$$



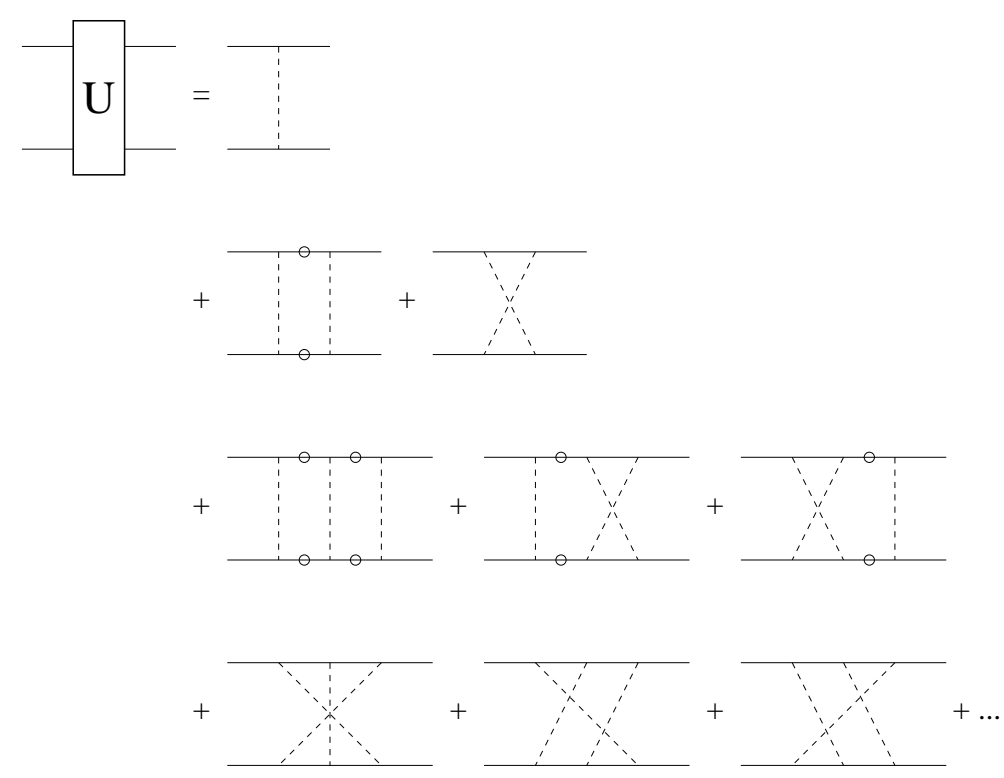

Figure 19: The quasipotential kernel series.

which implies that $U^{(n)}$ is given by

$$
U^{(n)}=V^{(n)}-\sum_{\substack{i, j>0 \\ i+j=n}} V^{(i)} \Delta G U^{(j)},
$$

or, equivalently,

$$
U^{(n)}=V^{(n)}-\sum_{\substack{i, j>0 \\ i+j=n}} U^{(i)} \Delta G V^{(j)}
$$

If we eliminate the presence of $U^{(j)}$ 's in the right hand side by consequent iterations, the answer will be given by the following series

$$
U^{(n)}=\sum_{k=1}^{n} \sum_{\substack{i_{0}, i_{1}, \ldots, i_{k-1}>0 \\ i_{0}+i_{1}+\ldots+i_{k-1}=n}} V^{\left(i_{0}\right)} \prod_{0<j<k}\left(-\Delta G V^{\left(i_{j}\right)}\right) .
$$

This terse expression has a clear meaning and can be readily illustrated by Fig. 19, where the first term represents $U^{(1)}$, terms on the second row 
represent $U^{(2)}$, and all terms on both the third and fourth rows are $U^{(3)}$. Circles indicate the appearances of $\Delta G$. We can see now that, in the OBE approximation, the Bethe-Salpeter and the quasipotential kernels are the same, $V^{(1)}=U^{(1)}$, and are given by the first diagram on Fig. 19, whereas $U^{(2)}$ differs from $V^{(2)}, U^{(2)}=V^{(2)}-V^{(1)} \Delta G V^{(1)}$. An $n$-boson-exchange contribution to the quasipotential kernel incorporates $i$-boson-exchange contributions to the Bethe-Salpeter kernel for all $i$ 's such that $1 \leq i \leq n$. In this sense, the quasipotential kernel is a resummation of the Bethe-Salpeter kernel.

\subsubsection{Current Expansion}

The next step is to demonstrate that the current expansion with respect to the number of boson exchanges does not interfere with the current conservation. Namely, we have to show that the diagram on Fig. 20 is commutative.

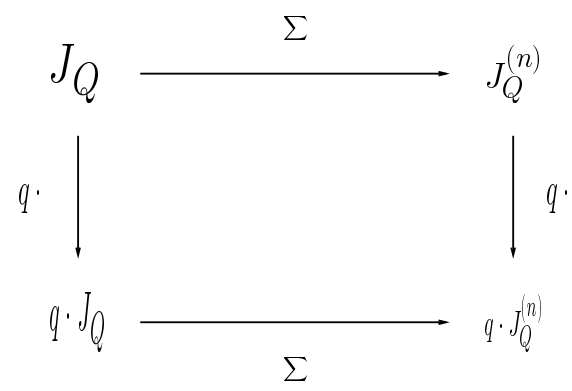

Figure 20: The divergence and expansion of the effective current.

We first consider $\left(q_{\mu} J_{Q}^{\mu}\right)^{(n)}$. We start with the effective current divergence $q_{\mu} J_{Q}^{\mu}$ given by (107). Rewriting the first term on the right hand side of (107) as

$$
-\mathcal{G}_{Q}^{-1} g G_{B S}^{-1} e_{\Sigma}(1-\Delta G U) \doteq-\mathcal{G}_{Q}^{-1} g G_{B S}^{-1} e_{\Sigma}
$$




$$
\begin{aligned}
& +\mathcal{G}_{Q}^{-1} g G_{B S}^{-1} e_{\Sigma} G_{B S} U \\
& -\mathcal{G}_{Q}^{-1} g G_{B S}^{-1} e_{\Sigma} g U
\end{aligned}
$$

we conclude that only the second term survives: the relative momentum to the left side of the charge operator is constrained, and it is impossible (due to the momentum conservation) to have the momentum constrained to the right side of the charge operator. Only the second term has the unconstrained momentum next to the charge operator. (The first term does not possess this property because of the notation convention about ".”" sign.) Performing analogous analysis of the second term of (107), we reduce (107) to

$$
q_{\mu} J_{Q}^{\mu} \doteq(1+U g) G_{B S}^{-1} e_{\Sigma} G_{B S} U-U G_{B S} e_{\Sigma} G_{B S}^{-1}(g U+1)
$$

where we have also used the explicit form of $\mathcal{G}_{Q}^{-1}$.

Alternatively, we can obtain the same answer directly from the generic expression for the impulse approximation part of the current (169). Indeed, consider $J_{\text {eff }, 1}^{\mu}$ first:

$$
J_{\mathrm{eff}, 1}^{\mu} \doteq-U G_{1} J_{1}^{\mu}-J_{1}^{\mu} G_{1} U-U G_{1} J_{1}^{\mu} g U-U g J_{1}^{\mu} G_{1} U-U G_{1} i J_{1}^{\mu} G_{1} G_{2} U
$$

Its divergence is equal to

$$
\begin{aligned}
q_{\mu} J_{\mathrm{eff}, 1}^{\mu} \doteq & -U G_{1} e_{1} G_{1}^{-1}-\left[e_{1}, U\right]+G_{1}^{-1} e_{1} G_{1} U \\
& -U G_{1} e_{1} G_{1}^{-1} g U+U\left[e_{1}, g\right] U+U g G_{1}^{-1} e_{1} G_{1} U G-U\left[e_{1}, G_{B S}\right] U \\
\doteq & (1+U g) G_{1}^{-1} e_{1} G_{1} U-U G_{1} e_{1} G_{1}^{-1}(g U+1) \\
& -\left[e_{1}, U\right]-U\left[e_{1}, \Delta G\right] U .
\end{aligned}
$$

The divergence of $J_{\text {eff, } 2}^{\mu}$ is given by the same expression with index $1 \rightarrow 2$. Since

$$
G_{B S}^{-1} e_{\Sigma} G_{B S}=i G_{1}^{-1} G_{2}^{-1}\left(e_{1}+e_{2}\right)(-i) G_{1} G_{2}
$$




$$
=G_{1}^{-1} e_{1} G_{1}+G_{2}^{-1} e_{2} G_{2},
$$

and similarly for $G_{B S} e_{\Sigma} G_{B S}^{-1}$, the sum of $q_{\mu} J_{\text {eff }, 1}^{\mu}$ and $q_{\mu} J_{\text {eff }, 2}^{\mu}$ can be written as

$$
\begin{aligned}
q_{\mu} J_{\mathrm{eff}, \mathrm{IA}}^{\mu} \doteq & (1+U g) G_{B S}^{-1} e_{\Sigma} G_{B S} U-U G_{B S} e_{\Sigma} G_{B S}^{-1}(g U+1) \\
& -\left[e_{\Sigma}, U\right]-U\left[e_{\Sigma}, \Delta G\right] U
\end{aligned}
$$

Adding to this the divergence of the exchange current which is given by the generic expression (141), we obtain (191).

When the kernel is expanded as in (185), the $n$-boson exchange contribution to the divergence of the current (191) follows directly from (191)

$$
\begin{aligned}
\left(q_{\mu} J_{Q}^{\mu}\right)^{(n)} \doteq & G_{B S}^{-1} e_{\Sigma} G_{B S} U^{(n)}-U^{(n)} G_{B S} e_{\Sigma} G_{B S}^{-1} \\
& +\sum_{\substack{i, j>0 \\
i+j=n}}\left(U^{(i)} g G_{B S}^{-1} e_{\Sigma} G_{B S} U^{(j)}\right. \\
& \left.-U^{(i)} G_{B S} e_{\Sigma} G_{B S}^{-1} g U^{(j)}\right), \quad n>0,
\end{aligned}
$$

where, if a term is linear with respect to $U$, we just replaced $U$ by $U^{(n)}$, and if it is quadratic, we picked up all possible contributions with the total number of boson exchanges being equal to $n$. In other words, we followed the down/right branch of Fig. 20 and obtained (196). We have to show now that the right/down branch lead to the same result.

The form of the $n$-boson exchange contribution to the effective current can also be directly derived from (104), where not only the kernel $U$ but also the current $J^{\mu}$ are the subject to expansion. A special case is

$$
J_{Q}^{\mu(0)} \doteq J^{\mu(0)} \doteq J_{\mathrm{IA}}^{\mu}
$$

which is not present because of the momentum conservation issues. Obviously, this is consistent with the elimination of the first term in (190). If we had 
kept this term, it would have produced the 0th order (no boson exchanges) contribution, $-G_{B S}^{-1} e_{\Sigma}$, and, hence,

$$
\left(q_{\mu} J_{Q}^{\mu}\right)^{(0)} \doteq\left[e_{\Sigma}, G_{B S}^{-1}\right] \doteq q_{\mu}\left(J_{Q}^{\mu(0)}\right)
$$

However, since we decided to put all terms violating the momentum conservation to zero, the integer $n$ is assumed to be greater than zero in

$$
\begin{aligned}
J_{Q}^{\mu(n)} \doteq & J_{\mathrm{ex}}^{\mu(n)}-U^{(n)} \Delta G J_{\mathrm{IA}}^{\mu}-J_{\mathrm{IA}}^{\mu} \Delta G U^{(n)} \\
& -\sum_{\substack{i, j>0 \\
i+j=n}}\left(U^{(i)} \Delta G J_{\mathrm{ex}}^{\mu(j)}+J_{\mathrm{ex}}^{\mu(i)} \Delta G U^{(j)}-U^{(i)} \Delta G J_{\mathrm{IA}}^{\mu} \Delta G U^{(j)}\right) \\
& +\sum_{\substack{i, j, k>0 \\
i+j+k=n}} U^{(i)} \Delta G J_{\mathrm{ex}}^{\mu(j)} \Delta G U^{(k)}, \quad n>0
\end{aligned}
$$

where the last term is cubic with respect to expansion. The use of the WardTakahashi identities gives

$$
\begin{aligned}
q_{\mu}\left(J_{Q}^{\mu(n)}\right) \doteq & {\left[e_{\Sigma}, V^{(n)}\right]-U^{(n)} \Delta G\left[e_{\Sigma}, G_{B S}^{-1}\right]-\left[e_{\Sigma}, G_{B S}^{-1}\right] \Delta G U^{(n)} } \\
& -\sum_{\substack{i, j>0 \\
i+j=n}}\left(U^{(i)} \Delta G\left[e_{\Sigma}, V^{(j)}\right]+\left[e_{\Sigma}, V^{(i)}\right] \Delta G U^{(j)}\right. \\
& \left.-U^{(i)} \Delta G\left[e_{\Sigma}, G_{B S}^{-1}\right] \Delta G U^{(j)}\right) \\
& +\sum_{\substack{i, j, k>0 \\
i+j+k=n}} U^{(i)} \Delta G\left[e_{\Sigma}, V^{(j)}\right] \Delta G U^{(k)} .
\end{aligned}
$$

If we now want to omit terms violating momentum conservation, we observe that the first term, all terms on the second row and the last term remain intact, whereas the second and the third terms should be put to

$$
\begin{aligned}
-U^{(n)} \Delta G\left[e_{\Sigma}, G_{B S}^{-1}\right] & \mapsto-U^{(n)} G_{B S}\left[e_{\Sigma}, G_{B S}^{-1}\right], \\
-\left[e_{\Sigma}, G_{B S}^{-1}\right] \Delta G U^{(n)} & \mapsto-\left[e_{\Sigma}, G_{B S}^{-1}\right] G_{B S} U^{(n)},
\end{aligned}
$$


and for the term on the third row of (200), we have

$$
\begin{aligned}
& -U^{(i)} \Delta G\left[e_{\Sigma}, G_{B S}^{-1}\right] \Delta G U^{(j)} \doteq-U^{(i)}\left(G_{B S}-g\right)\left[e_{\Sigma}, G_{B S}^{-1}\right]\left(G_{B S}-g\right) U^{(j)} \\
\mapsto & -U^{(i)} G_{B S}\left[e_{\Sigma}, G_{B S}^{-1}\right] G_{B S} U^{(j)}+U^{(i)} G_{B S}\left[e_{\Sigma}, G_{B S}^{-1}\right] g U^{(j)} \\
& +U^{(i)} g\left[e_{\Sigma}, G_{B S}^{-1}\right] G_{B S} U^{(j)} \\
\doteq & U^{(i)}\left[e_{\Sigma}, G_{B S}\right] U^{(j)}+U^{(i)}\left(G_{B S} e_{\Sigma} G_{B S}^{-1} g-e_{\Sigma} g\right) U^{(j)} \\
& +U^{(i)}\left(g e_{\Sigma}-g G_{B S}^{-1} e_{\Sigma} G_{B S}\right) U^{(j)} \\
\doteq & U^{(i)}\left[e_{\Sigma}, \Delta G\right] U^{(j)}+U^{(i)}\left(G_{B S} e_{\Sigma} G_{B S}^{-1} g-g G_{B S}^{-1} e_{\Sigma} G_{B S}\right) U^{(j)}
\end{aligned}
$$

Collecting results from (201), (202) and (203) and substituting the explicit form of all commutators into (200), we write

$$
\begin{gathered}
q_{\mu}\left(J_{Q}^{\mu(n)} \doteq e_{\Sigma} V^{(n)}-V^{(n)} e_{\Sigma}-U^{(n)} G_{B S} e_{\Sigma} G_{B S}^{-1}+U^{(n)} e_{\Sigma}\right. \\
-e_{\Sigma} U^{(n)}+G_{B S}^{-1} e_{\Sigma} G_{B S} U^{(n)} \\
-\sum_{\substack{i, j>0 \\
i+j=n}}\left(U^{(i)} \Delta G e_{\Sigma} V^{(j)}-U^{(i)} \Delta G V^{(j)} e_{\Sigma}\right. \\
+e_{\Sigma} V^{(i)} \Delta G U^{(j)}-V^{(i)} e_{\Sigma} \Delta G U^{(j)} \\
+U^{(i)} e_{\Sigma} \Delta G U^{(j)}-U^{(i)} \Delta G e_{\Sigma} U^{(j)} \\
\left.+U^{(i)} G_{B S} e_{\Sigma} G_{B S}^{-1} g U^{(j)}-U^{(i)} g G_{B S}^{-1} e_{\Sigma} G_{B S} U^{(j)}\right) \\
+\sum_{\substack{i, j, k>0 \\
i+j+k=n}}^{\left(U^{(i)} \Delta G e_{\Sigma} V^{(j)} \Delta G U^{(k)}\right.} \\
\left.\quad-U^{(i)} \Delta G V^{(j)} e_{\Sigma} \Delta G U^{(k)}\right) .
\end{gathered}
$$

To alleviate the navigation from the last to the next expressions, we produce a table that maps the row and the number of a term in that row in (204) to (number,row) pairs of the same term in (205). Using this table, we conclude, for example, that the first (and the only) term on the eighth row of (204) 
Table 2: (204) $\mapsto(205)$ transformation.

\begin{tabular}{|c||c|c|c|c|c|c|c|c|}
\hline & r1 & r2 & r3 & r4 & r5 & r6 & r7 & r8 \\
\hline \hline n1 & n1,r1 & n3,r1 & n1,r4 & n2,r1 & n3,r5 & n1,r7 & n2,r4 & n2,r5 \\
\hline n2 & n1,r2 & n1,r3 & n2,r2 & n1,r5 & n3,r4 & n1,r6 & - & - \\
\hline n3 & n2,r3 & - & - & - & - & - & - & - \\
\hline n4 & n3,r2 & - & - & - & - & - & - & - \\
\hline
\end{tabular}

migrates to the second position in the fifth row of (205), where we also change some of summation indices:

$$
\begin{aligned}
q_{\mu}\left(J_{Q}^{\mu(n)}\right) \doteq & e_{\Sigma}\left(V^{(n)}-\sum_{\substack{i, j>0 \\
i+j=n}} V^{(i)} \Delta G U^{(j)}-U^{(n)}\right) \\
& -\left(V^{(n)}-\sum_{\substack{i, j>0 \\
i+j=n}} U^{(i)} \Delta G V^{(j)}-U^{(n)}\right) e_{\Sigma} \\
& +G_{B S}^{-1} e_{\Sigma} G_{B S} U^{(n)}-U^{(n)} G_{B S} e_{\Sigma} G_{B S}^{-1} \\
& -\sum_{\substack{i, j>0 \\
i+j=n}} U^{(i)} \Delta G e_{\Sigma}\left(V^{(j)}-\sum_{\substack{p, q>0 \\
p+q=j}} V^{(p)} \Delta G U^{(q)}-U^{(j)}\right) \\
& +\sum_{\substack{i, j>0 \\
i+j=n}}\left(V^{(i)}-\sum_{\substack{p, q>0 \\
p+q=i}} U^{(p)} \Delta G V^{(q)}-U^{(i)}\right) e_{\Sigma} \Delta G U^{(j)} \\
& +\sum_{\substack{i, j>0 \\
i+j=n}}\left(U^{(i)} g G_{B S}^{-1} e_{\Sigma} G_{B S} U^{(j)}\right. \\
& \left.-U^{(i)} G_{B S} e_{\Sigma} G_{B S}^{-1} g U^{(j)}\right) .
\end{aligned}
$$

The use of the equations for the $n$-boson contribution to the quasipotential kernel in the form of (187) and (188) eliminates all terms on the first, second, fourth and fifth rows, and what remains (terms on the third row and the last 
two terms) coincides with equation (196). This means that

$$
q_{\mu}\left(J_{Q}^{\mu(n)}\right) \doteq\left(q_{\mu} J_{Q}^{\mu}\right)^{(n)}
$$

the diagram on Fig. 20 is commutative, and the expansion procedure does not interfere with the proof of the current conservation since all contributions can be considered independently.

\subsubsection{Current Truncation}

Unfortunately, as soon as truncation is in effect $\left(\sum_{n>0} \rightarrow \sum_{n>0}^{N}\right)$, equation (196) no longer corresponds to the conserved part of the effective current since it is essentially quadratic (all cubic terms vanish) with respect to $U$. Therefore, to restore the current conservation, we have to work out a new summation pattern and to give a reasonable interpretation of terms we have to add or subtract.

For this end, we first bring equation (191) to a more symmetric form. It can be rewritten as

$$
q_{\mu} J_{Q}^{\mu} \doteq\left(g^{-1}+\cdot U \cdot\right) \hat{O}_{L} U \cdot-\cdot U \hat{O}_{R}\left(g^{-1}+\cdot U \cdot\right)
$$

where we explicitly keep track of the relative momentum constraints around $U$ and where

$$
\begin{aligned}
& \hat{O}_{L}=g G_{B S}^{-1} e_{\Sigma} G_{B S}, \\
& \hat{O}_{R}=G_{B S} e_{\Sigma} G_{B S}^{-1} g .
\end{aligned}
$$

We now want to add four extra terms to (207). Having the relative momentum constrained to both side of the charge operator, all these terms violate 
momentum conservation and, therefore, after actual calculations should be always put to zero. Equation (207) becomes

$$
q_{\mu} J_{Q}^{\mu} \doteq\left(g^{-1}+\cdot U \cdot\right) \hat{O}_{L}\left(g^{-1}+U \cdot\right)-\left(g^{-1}+\cdot U\right) \hat{O}_{R}\left(g^{-1}+\cdot U \cdot\right)
$$

and it can not be rewritten as

$$
q_{\mu} J_{Q}^{\mu} \doteq \mathcal{G}_{Q}^{-1} \hat{O}_{L} \mathcal{G}_{Q}^{-1}-\mathcal{G}_{Q}^{-1} \hat{O}_{R} \mathcal{G}_{Q}^{-1}
$$

since

$$
\mathcal{G}_{Q}^{-1} \doteq g^{-1}+U=g^{-1}+\cdot U \cdot
$$

which is not the case for all terms in (207).

However, equation (207) possesses the necessary symmetry. Namely, it does have $\mathcal{G}_{Q}^{-1}$ to the left of $\hat{O}_{L}$ and to the right of $\hat{O}_{R}$. This property guarantees the conservation of the current. If we now expand $U$ as in (185) without keeping track of the momentum constraints and put, similar to (183),

$$
U^{(0)}=g^{-1}
$$

then equation (210) can be rewritten as

$$
q_{\mu} J_{Q}^{\mu} \doteq \sum_{i, j \geq 0} U^{(i)} \hat{O} U^{(j)}
$$

where

$$
\hat{O}=\hat{O}_{L}-\hat{O}_{R}
$$

The constraint conditions in (214) are properly taken care of by the structure of $\hat{O}$.

The form of the expression (214) extremely simplifies the following considerations. First, if we represent every term in (214) as a dot on Fig. 21, then 


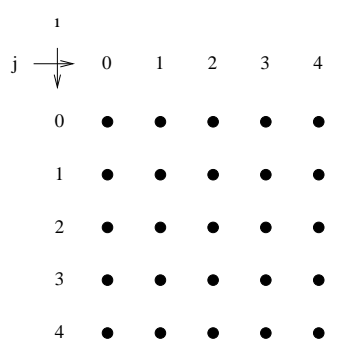

Figure 21: No truncation.

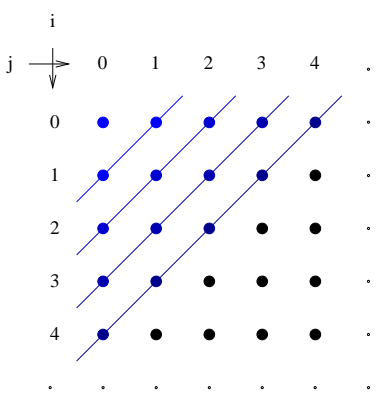

Figure 22: The $n$-boson exchange contributions. 
the exact answer (no truncation) is equivalent to keeping the infinite number of all dots on Fig. 21.

Second, we immediately see that the $n$-boson exchange contributions to (214) are represented by dots on the diagonal lines drawn on Fig.22. These contributions are given by

$$
q_{\mu} J_{Q}^{\mu(n)} \doteq \sum_{\substack{i, j \geq 0 \\ i+j=n}} U^{(i)} \hat{O} U^{(j)}
$$

which, after omitting a number of zero terms, produces (196). Indeed,

$$
\begin{aligned}
q_{\mu} J_{Q}^{\mu(n)} \doteq & \sum_{i=0}^{n} U^{(i)} \hat{O} U^{(n-i)} \doteq U^{(0)} \hat{O} U^{(n)}+U^{(n)} \hat{O} U^{(0)}+\sum_{i=1}^{n-1} U^{(i)} \hat{O} U^{(n-i)} \\
\doteq & g^{-1} \hat{O} U^{(n)}+U^{(n)} \hat{O} g^{-1}+\sum_{\substack{i, j>0 \\
i+j=n}} U^{(i)} \hat{O} U^{(j)} \\
\doteq & g^{-1}\left(g G_{B S}^{-1} e_{\Sigma} G_{B S}-G_{B S} e_{\Sigma} G_{B S}^{-1} g\right) U^{(n)} \\
& U^{(n)}\left(g G_{B S}^{-1} e_{\Sigma} G_{B S}-G_{B S} e_{\Sigma} G_{B S}^{-1} g\right) g^{-1} \\
& +\sum_{\substack{i, j>0 \\
i+j=n}} U^{(i)}\left(g G_{B S}^{-1} e_{\Sigma} G_{B S}-G_{B S} e_{\Sigma} G_{B S}^{-1} g\right) U^{(j)} \\
\mapsto & G_{B S}^{-1} e_{\Sigma} G_{B S} U^{(n)}-U^{(n)} G_{B S} e_{\Sigma} G_{B S}^{-1} \\
& +\sum_{\substack{i, j>0 \\
i+j=n}}\left(U^{(i)} g G_{B S}^{-1} e_{\Sigma} G_{B S} U^{(j)}-U^{(i)} G_{B S} e_{\Sigma} G_{B S}^{-1} g U^{(j)}\right) .(217)
\end{aligned}
$$

We can truncate the series represented by Fig. 21 in a number of different ways. Any truncation technique should correspond to some pattern of figures covering certain number of dots. Any figure in a pattern is characterized by the scale factor, which is, of course, the level of truncation, $N$. When $N \rightarrow \infty$, 


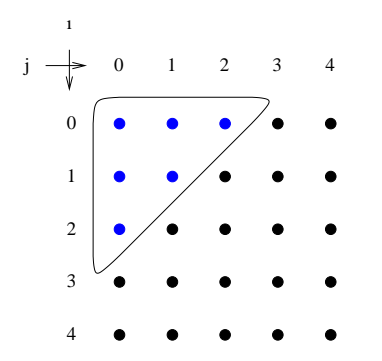

Figure 23: Up-to- $N$-boson exchange truncation.

the figure expands to cover all dots on Fig. 21. Looking at Fig. 22, we conclude that the pattern corresponding to the up-to- $N$-boson exchange truncation technique, defining the divergence of up-to- $N$-boson exchange current as

$$
q_{\mu} J_{Q}^{\mu}{ }^{[N]} \doteq q_{\mu} \sum_{n \geq 0}^{N} J_{Q}^{\mu(n)} \doteq \sum_{i, j \geq 0}^{i+j \leq N} U^{(i)} \hat{O} U^{(j)},
$$

is a pattern of triangles shown on Fig. 23, where $N=2$. As we saw, this pattern does not provide for the current conservation.

A truncation pattern complying with the current conservation can be easily found if we rewrite the truncated form of the quasipotential wave equation as

$$
<\psi_{Q}^{[N]}\left|U^{[N]}=U^{[N]}\right| \psi_{Q}^{[N]}>=0,
$$

where

$$
U^{[N]}=\sum_{n \geq 0}^{N} U^{(n)} .
$$

Obviously, the pattern of squares on Fig. $24(N=2)$ corresponds to the truncation technique restoring the current conservation. The divergence of the current truncated as on Fig. 24 is given by

$$
q_{\mu} \widetilde{J}_{Q}^{\mu[N]} \doteq U^{[N]} \hat{O} U^{[N]} .
$$




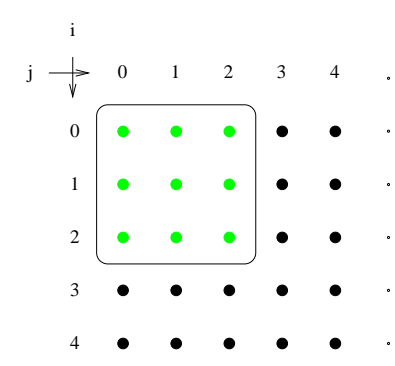

Figure 24: Current is conserved.

The difference between currents $\tilde{J}_{Q}^{\mu[N]}$ and $J_{Q}^{\mu[N]}, D_{Q}^{\mu[N]}$,

$$
\tilde{J}_{Q}^{\mu[N]} \doteq J_{Q}^{\mu[N]}+D_{Q}^{\mu[N]},
$$

will be discussed in a moment.

The wave equation in the form of (219) is not perfectly correct because of the momentum constraint issues discussed above. The correct form of the wave equation is

$$
<\psi_{Q}^{[N]}\left|\mathcal{G}_{Q}^{-1[N]} \doteq \mathcal{G}_{Q}^{-1[N]}\right| \psi_{Q}^{[N]}>\doteq 0,
$$

where

$$
\mathcal{G}_{Q}^{-1[N]} \doteq g^{-1}+\sum_{n>0}^{N} U^{(n)}
$$

However, a more rigorous check that the current (221) is conserved can be easily done.

Indeed,

$$
\begin{aligned}
q_{\mu} \tilde{J}_{Q}^{\mu[N]} & \doteq U^{[N]} \hat{O} U^{[N]} \doteq \sum_{i \geq 0}^{N} U^{(i)} \hat{O} \sum_{j \geq 0}^{N} U^{(j)} \\
& \doteq\left(g^{-1}+\sum_{i>0}^{N} U^{(i)}\right) \hat{O}_{L}\left(g^{-1}+\sum_{j>0}^{N} U^{(j)}\right)
\end{aligned}
$$




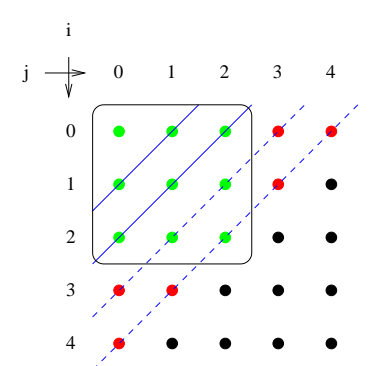

Figure 25: The interpretation of the current $J_{Q}^{\mu[N]}$.

$$
\begin{aligned}
& -\left(g^{-1}+\sum_{i>0}^{N} U^{(i)}\right) \hat{O}_{R}\left(g^{-1}+\sum_{j>0}^{N} U^{(j)}\right) \\
\doteq & \left(g^{-1}+\sum_{i>0}^{N} U^{(i)}\right) g G_{B S}^{-1} e_{\Sigma} G_{B S}\left(g^{-1}+\sum_{j>0}^{N} U^{(j)}\right) \\
& -\left(g^{-1}+\sum_{i>0}^{N} U^{(i)}\right) G_{B S} e_{\Sigma} G_{B S}^{-1} g\left(g^{-1}+\sum_{j>0}^{N} U^{(j)}\right) \\
\mapsto \quad & \left(g^{-1}+\sum_{i>0}^{N} U^{(i)}\right) g G_{B S}^{-1} e_{\Sigma} G_{B S} \sum_{j>0}^{N} U^{(j)} \\
& -\sum_{i>0}^{N} U^{(i)} G_{B S} e_{\Sigma} G_{B S}^{-1} g\left(g^{-1}+\sum_{j>0}^{N} U^{(j)}\right) \\
\doteq & \mathcal{G}_{Q}^{-1}[N] \hat{O}_{L} \sum_{j>0}^{N} U^{(j)}-\sum_{i>0}^{N} U^{(i)} \hat{O}_{R} \mathcal{G}_{Q}^{-1[N]},
\end{aligned}
$$

where the " $\mapsto$ " sign again indicates that all terms violating the momentum conservation are eliminated. Equation (225) along with the wave equation (223) guarantees that the current

$$
\tilde{\mathcal{J}}_{Q}^{\mu[N]}=<\psi_{Q}^{[N]}\left|\tilde{J}_{Q}^{\mu[N]}\right| \psi_{Q}^{[N]}>
$$

is conserved.

Given the set of Figs. 21-24, the interpretation of the current $D_{Q}^{\mu[N]}$ (or, 
effectively, $\tilde{J}_{Q}^{\mu}{ }^{[N]}$ ) is rather straightforward and is illustrated by Fig. 25, where the case of $N=2$ is considered. First, we note that the divergence of the upto- $N$-boson exchange current $J_{Q}^{\mu[N]}$ is represented by dots lying on the solid lines. To restore the current conservation we had to add contributions coming from up-to- $2 N$-boson exchanges, $D_{Q}^{\mu[N]}$, represented by dots lying on dashed lines and inside the square. The result is current $\widetilde{J}_{Q}^{\mu[N]}$ (all dots inside the square). However, if we added all contributions from $J_{Q}^{\mu(n)}$ with $N<n \leq 2 N$, this would bring some extraneous terms represented by dots on dashed lines and outside the square. These extraneous contributions are denoted as $S_{Q}^{\mu[N]}$ below, and the expression for the current $D_{Q}^{\mu[N]}$ becomes

$$
D_{Q}^{\mu[N]} \doteq \sum_{n>N}^{2 N} J_{Q}^{\mu(n)}-S_{Q}^{\mu[N]}
$$

or, for $\widetilde{J}_{Q}^{\mu[N]}$,

$$
\tilde{J}_{Q}^{\mu[N]} \doteq \sum_{n \geq 0}^{2 N} J_{Q}^{\mu(n)}-S_{Q}^{\mu[N]},
$$

where the longitudinal part of the current we have to subtract is given by

$$
S_{Q}^{\mu[N]} \doteq \frac{q^{\mu}}{q^{2}} \sum_{\substack{i \mid j>N \\ i+j \leq 2 N}} U^{(i)} \hat{O} U^{(j)} .
$$

The fact that the conserved part of the current incorporates contributions coming from up-to- $2 \mathrm{~N}$-boson exchanges is obviously explained by the presence of quadratic (with respect to the kernel) terms in the expression for the divergence of the current. If this expression had contained cubic terms, then Fig. 21 would have been three-dimensional and we would have had to add contributions from up-to- $3 \mathrm{~N}$-boson exchange parts of the current. 


\subsubsection{OBE Example}

And, finally, we consider the simplest example of the OBE approximation. The conserved part of the current is equal in this case to

$$
\tilde{J}_{Q}^{\mu[1]} \doteq \sum_{n=0}^{2} J_{Q}^{\mu(n)}-S_{Q}^{\mu[1]} .
$$

The 0th order contribution

$$
J_{Q}^{\mu(0)} \doteq J_{\mathrm{IA}}^{\mu} \mapsto 0
$$

as already discussed. For the one-boson exchange current, we have

$$
J_{Q}^{\mu(1)} \doteq-J_{\mathrm{IA}}^{\mu} \Delta G V-V \Delta G J_{\mathrm{IA}}^{\mu}+J_{\mathrm{ex}}^{\mu}
$$

where

$$
V=V^{(1)}=U^{(1)}
$$

and

$$
J_{\mathrm{ex}}^{\mu} \equiv J_{\mathrm{ex}}^{\mu(1)}
$$

The divergence of $J_{Q}^{\mu(1)}$ is

$$
\begin{aligned}
q_{\mu} J_{Q}^{\mu(1)} & \doteq-\left[e_{\Sigma}, G_{B S}^{-1}\right]\left(G_{B S}-g\right) V-V\left(G_{B S}-g\right)\left[e_{\Sigma}, G_{B S}^{-1}\right]+\left[e_{\Sigma}, V\right] \\
& \mapsto G_{B S}^{-1} e_{\Sigma} G_{B S} V-V G_{B S} e_{\Sigma} G_{B S}^{-1} .
\end{aligned}
$$

The two-boson exchange current is somewhat more complicated

$$
\begin{aligned}
J_{Q}^{\mu(2) \doteq} & -J_{\mathrm{IA}}^{\mu} \Delta G U^{(2)}-U^{(2)} \Delta G J_{\mathrm{IA}}^{\mu}+J_{\mathrm{ex}}^{\mu(2)} \\
& -J_{\mathrm{ex}}^{\mu} \Delta G V-V \Delta G J_{\mathrm{ex}}^{\mu}+V \Delta G J_{\mathrm{IA}}^{\mu} \Delta G V .
\end{aligned}
$$

Its divergence is given by

$$
q_{\mu} J_{Q}^{\mu(2)} \doteq-\left[e_{\Sigma}, G_{B S}^{-1}\right]\left(G_{B S}-g\right) U^{(2)}-U^{(2)}\left(G_{B S}-g\right)\left[e_{\Sigma}, G_{B S}^{-1}\right]+\left[e_{\Sigma}, V^{(2)}\right]
$$




$$
\begin{aligned}
& -\left[e_{\Sigma}, V\right] \Delta G V-V \Delta G\left[e_{\Sigma}, V\right]+V \Delta G\left[e_{\Sigma}, G_{B S}^{-1}\right] \Delta G V \\
\mapsto \quad & G_{B S}^{-1} e_{\Sigma} G_{B S} U^{(2)}-U^{(2)} G_{B S} e_{\Sigma} G_{B S}^{-1} \\
& -e_{\Sigma} U^{(2)}+U^{(2)} e_{\Sigma}+e_{\Sigma} V^{(2)}-V^{(2)} e_{\Sigma} \\
& -e_{\Sigma} V \Delta G V+V e_{\Sigma} \Delta G V-V \Delta G e_{\Sigma} V+V \Delta G V e_{\Sigma} \\
& +V g G_{B S}^{-1} e_{\Sigma} G_{B S} V-V G_{B S} e_{\Sigma} G_{B S}^{-1} g V-V e_{\Sigma} \Delta G V+V \Delta G e_{\Sigma} V \\
\doteq \quad & G_{B S}^{-1} e_{\Sigma} G_{B S} U^{(2)}-U^{(2)} G_{B S} e_{\Sigma} G_{B S}^{-1} \\
& +V g G_{B S}^{-1} e_{\Sigma} G_{B S} V-V G_{B S} e_{\Sigma} G_{B S}^{-1} g V,
\end{aligned}
$$

where, at the last step, we used

$$
U^{(2)}=V^{(2)}-V \Delta G V
$$

The current we have to subtract is

$$
S_{Q}^{\mu[1]} \doteq \frac{q^{\mu}}{q^{2}}\left(g^{-1} \hat{O} U^{(2)}+U^{(2)} \hat{O} g^{-1}\right)
$$

For its divergence, we have

$$
\begin{aligned}
& q_{\mu} S_{Q}^{\mu[1] \doteq} g^{-1}\left(g G_{B S}^{-1} e_{\Sigma} G_{B S}-G_{B S} e_{\Sigma} G_{B S}^{-1} g\right) U^{(2)} \\
& U^{(2)}\left(g G_{B S}^{-1} e_{\Sigma} G_{B S}-G_{B S} e_{\Sigma} G_{B S}^{-1} g\right) g^{-1} \\
& \mapsto G_{B S}^{-1} e_{\Sigma} G_{B S} U^{(2)}-U^{(2)} G_{B S} e_{\Sigma} G_{B S}^{-1} .
\end{aligned}
$$

Collecting results from (235), (237) and (240), we obtain the following expression for the divergence of the conserved part of the effective current in the OBE approximation:

$$
\begin{aligned}
q_{\mu} \tilde{J}_{Q}^{\mu[1] \doteq} & G_{B S}^{-1} e_{\Sigma} G_{B S} V-V G_{B S} e_{\Sigma} G_{B S}^{-1} \\
& +V g G_{B S}^{-1} e_{\Sigma} G_{B S} V-V G_{B S} e_{\Sigma} G_{B S}^{-1} g V \\
\doteq & \left(g^{-1}+V\right) \hat{O}_{L} V-V \hat{O}_{R}\left(g^{-1}+V\right),
\end{aligned}
$$


which reproduces (225) with $N=1$. Since the wave equation in the OBE approximation has the form

$$
<\psi_{Q}^{[1]}\left|\left(g^{-1}+V\right) \doteq\left(g^{-1}+V\right)\right| \psi_{Q}^{[1]}>\doteq 0
$$

the current

$$
\tilde{\mathcal{J}}_{Q}^{\mu[1]}=<\psi_{Q}^{[1]}\left|\tilde{J}_{Q}^{\mu[1]}\right| \psi_{Q}^{[1]}>
$$

is conserved. 


\section{Chapter 6}

\section{Conclusion and Discussions}

We extended the Mandelstam formalism to the generic quasipotential approach. In this formalism, the quasipotential theory has the same form as the Bethe-Salpeter theory in the original Mandelstam formalism. This is another clear indication that any quasipotential equation is equivalent to the Bethe-Salpeter equation.

The new quasipotential Mandelstam formalism provides all necessary tools to construct the generic effective current operator. As expected, the matrix elements of this current are equal to the Bethe-Salpeter effective current matrix elements. The conservation of the current is checked using the Ward-Takahashi identities.

The divergence of the quasipotential effective current is essentially quadratic with respect to the quasipotential kernel. Therefore, some additional technique is required in order to retain the current conservation after truncation is performed. We gave an explicit interpretation of contributions one had to add or subtract to extract the conserved part of the current at different levels of boson exchanges. 
And, finally, we should emphasize an importance of the observation made on page 57. Rather straightforward and transparent field theoretical considerations allow us to start with the Bethe-Salpeter effective current and to transform it to the quasipotential effective current using the Bethe-Salpeter equation for the scattering matrix in such a way that the resultant current satisfy the following three requirements:

- The current should produce the same matrix elements as the BetheSalpeter current. As soon as the procedure uses the Bethe-Salpeter equation only, this requirement is always satisfied.

- The current should be defined on the submanifold, to which the phase space of the system is reduced. This means that the initial and the final momenta should be properly constrained. We saw that an attempt to "minimize" the current in the off-shell cases (Fig. 17) led to a current that did not satisfy this requirement.

- The current should be conserved. Practically, this means that the divergence of the current must be given in terms of $\mathcal{G}_{Q}^{-1} \mathcal{O}$ and $\mathcal{O G}_{Q}^{-1}$, where $\mathcal{O}$ is some operator string not having singularities that would be able to cancel zeros of $\mathcal{G}_{Q}^{-1}$. If we used the current $J_{\mathrm{G}, 2}^{\mu}$ in Section 4.2 as $-U G_{1} G_{2} i J_{2}^{\mu} G_{2} U$, then the divergence of $J_{\mathrm{G}, 2}^{\mu}$ would be given by $-U\left[e_{2}, G_{B S}\right] U$. When summed with the divergence of the exchange part of the current, $\left[e_{2}, U\right]+U\left[e_{2}, \Delta G\right] U$, this would give the divergence of the total effective current as $\left[e_{2}, U\right]-U\left[e_{2}, g_{G}\right] U$, which could not be rewritten as $\mathcal{G}_{G}^{-1} g_{G} e_{2} U-U e_{2} g_{G} \mathcal{G}_{G}^{-1}$ since zeros in $\mathcal{G}_{G}^{-1}$ should be first canceled by singularities in $g_{G}$, and, hence, the current would not be conserved. The situation would not be cured by 
our also using $J_{\mathrm{G}, \mathrm{ex}, 2}^{\mu}$ as $U G_{B S} J_{\mathrm{ex}, 2}^{\mu} G_{B S} U$ (the simpliest form of the current in the sense that we just turned $<\Gamma\left|G_{B S}\left(J_{\mathrm{IA}, 2}^{\mu}+J_{\mathrm{ex}, 2}^{\mu}\right) G_{B S}\right| \Gamma>$ into $\left.<\Gamma\left|g_{G} U G_{B S}\left(J_{\mathrm{IA}, 2}^{\mu}+J_{\mathrm{ex}, 2}^{\mu}\right) G_{B S} U g_{G}\right| \Gamma>\right)$. The divergence of $J_{\mathrm{G}, \mathrm{ex}, 2}^{\mu}$ would be given by $U G_{B S}\left[e_{2}, V\right] G_{B S} U$, and summing this with the divergence of $J_{\mathrm{G}, 2}^{\mu}$, we would obtain the total divergence as $U G_{B S}\left[e_{2}, \mathcal{G}^{-1}\right] G_{B S} U$, and the current would not be conserved either.

As shown in Section 4.2, one can obtain a number of different currents satisfying these three requirements. Through the successive iterations using the Bethe-Salpeter equation, one can end up with more and more complicated results. The Mandelstam formalism is characterized by the fact that, being a strict field theoretical result, it delivers the "minimal" answer. 


\section{Bibliography}

[1] G. E. Brown and A. D. Jackson, The Nucleon-Nucleon Interaction (North-Holland, Amsterdam, 1976).

[2] CEBAF experiments 89-012, 89-028, 91-026, 93-038, 94-018, 96-003.

[3] E. E. Salpeter and H. A. Bethe, Phys. Rev. 84, 1232 (1951).

[4] F. Gross, Relativistic Quantum Mechanics and Field Theory (WileyInterscience, New York, 1993).

[5] T. Nieuwenhuis and J. A. Tjon, Phys. Rev. Lett. 77, 814 (1996).

[6] Yu. A. Simonov and J. A. Tjon, Ann. Phys. 228, 1 (1993).

[7] K. Kusaka, K. Simpson and A. G. Williams, preprint hep-ph/9705298.

[8] J. Fleischer and J. A. Tjon, Nucl. Phys. B84, 375 (1975); Phys. Rev. D 15, 2537 (1977); 21, 87 (1980).

[9] E. van Faassen and J. A. Tjon, Phys. Rev. C 28, 2354 (1983); 30, 285 (1984); 33, 2105 (1986).

[10] M. J. Zuilhof and J. A. Tjon, Phys. Rev. C 22, 2369 (1980); 24, 736 (1981). 
[11] A. Yu. Umnikov and F. C. Khanna, Phys. Rev. C 49, 2311 (1994).

[12] S. Mandelstam, Proc. Roy. Soc. A 233, 248 (1955).

[13] J. C. Ward, Phys. Rev. 78, 182 (1950); Y. Takahashi, Nuovo Cimento 6, 371 (1957).

[14] W. Bentz, Nucl. Phys. A446, 678 (1985).

[15] N. K. Devine and S. J. Wallace, Phys. Rev. C 48, 973 (1993).

[16] J. Adam, Jr., J. W. Van Orden and F. Gross, preprint nucl-th/9710009.

[17] D. R. Phillips and S. J. Wallace, Phys. Rev. C 54, 507 (1996).

[18] M. Gell-Mann and F. Low, Phys. Rev. 84, 350, (1951).

[19] D. Lurie, Particles and Fields, (Wiley-Interscience, London, 1968).

[20] G. E. Shilov, Linear Algebra, (Prentice-Hall, New York, 1971).

[21] F. Gross and D. O. Riska, Phys. Rev. C 36, 1928 (1987).

[22] J. Adam, Jr., F. Gross, Ç. Şavkli and J. W. Van Orden, Phys. Rev. C 56, 641 (1997).

[23] F. Gross, J. W. Van Orden and K. Holinde, Phys. Rev. C 45, 2094 (1992).

[24] J. W. Van Orden, Czech J. Phys. 45, 181 (1995).

[25] R. Blankenbecler and R. Sugar, Phys. Rev. 142, 1051 (1966); A. A. Logunov and A. N. Tavkhelidze, Nuovo Cimento 29, 380 (1963).

[26] K. Erkelenz and K. Holide, Nucl. Phys. A194, 161 (1972). 
[27] I. T. Todorov, Phys. Rev. D 10, 2351 (1971).

[28] V. G. Kadychevsky, Nucl. Phys. B6, 125 (1968).

[29] R. H. Thompson, Phys. Rev. D 1, 1738 (1970).

[30] F. Gross, Phys. Rev. 186, 1448 (1969); Phys. Rev. D 10, 223 (1974).

[31] F. Gross, Phys. Rev. C 26, 2203 (1982).

[32] A. Klein and T.-S. H. Lee, Phys. Rev. D 10, 4308 (1974).

[33] V. B. Mandelzweig and S. J. Wallace, Phys. Lett. B. 197, 469 (1987).

[34] S. J. Wallace and V. B. Mandelzweig, Nucl. Phys. A503, 673 (1989).

[35] D. R. Phillips and S. J. Wallace, preprint nucl-th/9708027.

[36] D. R. Phillips, S. J. Wallace and N. K. Devine, preprint nucl-th/9802067.

[37] F. Coester and D. O. Riska, Annals Phys. 234, 141 (1994).

[38] L. Müller and W. Glökle, Nucl. Phys. B146, 393 (1978). 


\section{Curriculum Vitae}

I was born in St.Petersburg in 1969. In 1987, after finishing a college with the advanced programs in Physics and Mathematics, I entered the Physics Department of St.Petersburg University. Projects I worked on in SPbU included the calculation of the structure constants of the exceptional Cartan groups, the reduction of the regular inclusions of simple complex Lie algebra representations, exactly solvable models, the quantum inverse scattering and the differential calculus on quantum groups. I graduated from $\mathrm{SPbU}$ with the Diploma (with Honor) degree in 1993. In 1994, I was enrolled in the Ph.D program in Physics at ODU. Since then, I have been working on the electromagnetic scattering problems in the quasipotential formalism in the relativistic theory of bound states. 